\title{
IL-27 gene therapy induces depletion of Tregs and enhances the efficacy of cancer immunotherapy
}

\author{
Jianmin Zhu, ${ }^{1}$ Jin-Qing Liu, ${ }^{2}$ Min Shi, ${ }^{1}$ Xinhua Cheng, ${ }^{1}$ Miao Ding, ${ }^{1}$ Jianchao C. Zhang, ${ }^{3}$ \\ Jonathan P. Davis, ${ }^{3}$ Sanjay Varikuti, ${ }^{2}$ Abhay R. Satoskar, ${ }^{2}$ Lanchun Lu, ${ }^{4}$ Xueliang Pan, ${ }^{5}$ Pan Zheng, ${ }^{6}$ \\ Yang Liu, ${ }^{6}$ and Xue-Feng Bai ${ }^{1,2}$ \\ 'Pediatric Translational Medicine Institute, Shanghai Children's Medical Center, School of Medicine, Shanghai Jiao Tong \\ University, Shanghai, China. ${ }^{2}$ Department of Pathology and Comprehensive Cancer Center, ${ }^{3}$ Department of Physiology and \\ Cell Biology, ${ }^{4}$ Department of Radiation Oncology, and ${ }^{5}$ Center for Biostatistics, Ohio State University, Columbus, \\ Ohio, USA. 'enter for Cancer and Immunology Research, Children's Research Institute, Children's National Medical \\ Center, Washington, DC, USA.
}

Tumor-induced expansion of Tregs is a significant obstacle to cancer immunotherapy. However, traditional approaches to deplete Tregs are often inefficient, provoking autoimmunity. We show here that administration of IL-27-expressing recombinant adeno-associated virus (AAV-IL-27) significantly inhibits tumor growth and enhances T cell responses in tumors. Strikingly, we found that AAV-IL-27 treatment causes rapid depletion of Tregs in peripheral blood, lymphoid organs, and - most pronouncedly - tumor microenvironment. AAV-IL-27-mediated Treg depletion is dependent on IL-27 receptor and Stat1 in Tregs and is a combined result of CD25 downregulation in Tregs and inhibition of IL-2 production by T cells. In combination with a CM-CSF vaccine, AAVIL-27 treatment not only induced nearly complete tumor rejection, but also resulted in amplified neoantigen-specific T cell responses. AAV-IL-27 also dramatically increased the efficacy of antiPD-1 therapy, presumably due to induction of PD-L1 in T cells and depletion of Tregs. Importantly, AAV-IL-27 therapy did not induce significant adverse events, partially due to its induction of IL-10. In a plasmacytoma mouse model, we found that IL-10 was required for AAV-IL-27-mediated tumor rejection. Thus, our study demonstrates the potential of AAV-IL-27 as an independent cancer therapeutic and as an efficient adjuvant for cancer immunotherapy.

Authorship note: JZ and JQL contributed equally to this work.

Conflict of interest: The authors have declared that no conflict of interest exists.

Submitted: November 17, 2017 Accepted: February 28, 2018 Published: April 5, 2018

Reference information: JCI Insight. 2018;3(7):e98745. https:// doi.org/10.1172/jci.insight.98745.

\section{Introduction}

Identification of tumor antigens in the 1990s (1) paved the way for detection of tumor antigen-specific T cells in cancer patients $(2,3)$. It was found that tumor-reactive $T$ cells coexisted with cancer cells in tumors and draining lymph nodes without causing tumor regression (3). At least 3 factors were suggested to be responsible. First, the expansion of tumor-specific T cells may not be optimal. This notion led to the development of a therapeutic cancer vaccine but with only limited success until recently, when neoantigen-based individual cancer vaccines were developed (4-6). Second, Tregs have been shown to be expanded in cancer patients and are enriched in cancer lesions (7). Depletion of Tregs has led to increased tumor immunity in mice $(8,9)$. In anti-CTLA-4 antibody-treated tumor-bearing mice, Treg depletion in tumor lesion correlates with therapeutic response $(10,11)$. However, the impact of anti-CTLA-4 antibody therapy is tempered by autoimmune side effects (12). Third, cancer-reactive T cells are functionally exhausted or anergic (2) in the tumor microenvironment (TME). Checkpoint blockade therapies such as anti-PD-1 therapy have shown therapeutic effects to different cancer types $(13,14)$. However, many cancer types are resistant to anti-PD-1 therapy, and for those that respond, the overall responding rates remain low (15). Thus, development of novel strategies to enhance the efficacy of cancer immunotherapy is highly desired.

IL-27 is an IL-12 family cytokine that is known to inhibit autoimmune Th17 and Th2 responses $(16,17)$. Accumulating evidence from animal studies has indicated that both endogenous $(18,19)$ and exogenous $(20-22)$ IL-27 inhibit tumor growth. A variety of mechanisms including enhancement of tumor-specific T cell responses $(20,21)$ have been proposed. Our previous study $(23)$ has revealed that IL-27 programs CD $8^{+}$effector T cells into 
a unique T effector phenotype featured by producing both IFN- $\gamma$ and IL-10, which not only enhances T cell survival in TME, but also promotes T cell memory. In addition, we have also found that endogenous IL-27 is also required for cancer vaccine response (24). The unique functions of IL-27 suggest that developing IL-27-based cancer immunotherapy may lead to enhancing tumor immunity while inhibiting autoimmunity.

Although the role of IL-27 in tumor immunity has been appreciated for more than 10 years, developing IL-27 into a therapeutic to enhance tumor immunity has not been well achieved (25). Recombinant adenoassociated virus vectors (rAAV) are quickly establishing themselves as highly versatile gene delivery agents for gene therapy. The relatively low immunogenicity and toxic effects make rAAV arguably the gene therapy vector of choice for human clinical trials (26). In this study, we used an AAV system to systemically deliver IL-27 (AAV-IL-27) to treat mice with tumors. We show here that administration of AAV-IL-27 significantly inhibited tumor growth and enhances T cell responses in tumors. Strikingly, we found that AAV-IL-27 treatment caused rapid depletion of Tregs in peripheral blood, lymphoid organs, and - most pronouncedly — in TME. AAVIL-27-mediated Treg depletion is dependent on IL-27 receptor and Stat1 in Tregs and is a combined result of CD25 downregulation in Tregs and inhibition of T cell IL-2 production. In combination with a GM-CSF vaccine, AAV-IL-27 treatment not only induced nearly complete tumor inhibition and long-term survival of mice, but also resulted in amplified tumor neoantigen-specific T cell responses. Moreover, we found that AAV-IL-27 also dramatically increased the efficacy of anti-PD-1 therapy. Importantly, AAV-IL-27 therapy did not induce significant adverse events, partially due to its induction of IL-10. In a plasmacytoma mouse model, we found that IL-10 was required for AAV-IL-27-mediated tumor rejection. Thus, our study demonstrates the potential of AAV-IL-27 as an independent cancer therapeutic and as an efficient adjuvant for cancer immunotherapy.

\section{Results}

IL-27 gene therapy inhibits tumor growth. To determine if IL-27 can be used as a potential cancer therapeutic, we generated rAAV vectors that express IL-27 (AAV-IL-27) and the control AAV vector (AAV-ctrl) without the IL-27 cDNA. I.m. injection of $2 \times 10^{11}$ DNase resistance particle (DRP)/mouse of AAV-IL-27 achieved high and sustained IL-27 production in the peripheral blood of mice (Figure 1A). We then tested if AAV-IL-27 can be used to treat mice with B16 melanoma. First, we injected B16.F10 cells $\left(2 \times 10^{5} /\right.$ mouse) into C57BL/6 mice s.c., and 4 days after tumor cell injection, mice were injected with AAVIL-27 or AAV-ctrl virus i.m. at a dose of $2 \times 10^{11}$ DRP/mouse. As shown in Figure 1B, while B16.F10 tumors grew progressively in AAV-ctrl virus-treated mice, their growths were significantly inhibited in AAV-IL-27-treated mice. However, AAV-IL-27 treatment failed to inhibit B16 tumor growth in $1127 \mathrm{ra}^{-1}$ (Figure 1C) and $\mathrm{Rag}^{-1_{-}^{-}}$(Figure 1D) mice, suggesting that the tumor-inhibiting effect was IL-27 specific and not directed to tumor cells, but rather through activation of host immune responses. We also injected B16. F10 cells into B6 mice i.v., and 4 days later, mice were treated with a single dose $\left(2 \times 10^{11} \mathrm{DRP} / \mathrm{mouse}\right)$ of AAV-IL-27 or AAV-ctrl virus i.m. As demonstrated in Figure 1E, mice receiving AAV-IL-27 treatment had significantly reduced tumor foci in the lungs compared with mice treated with AAV-ctrl virus. Correspondingly, the lung weights of the AAV-IL-27-treated mice were significantly reduced. Similarly, we found that AAV-IL-27 therapy was also effective in inhibiting the growth of MC38 colon tumors (Figure $1 F$ ) and EO771 breast tumors (Figure 1G) in C57BL/6 mice, and of J558 plasmacytoma tumors (Figure $1 \mathrm{H})$ in BALB/c mice. Thus, AAV-IL-27 is an effective immunotherapeutic that inhibits the growth of a broad spectrum of cancer types in experimental mouse tumor models.

AAV-IL-27 therapy induces depletion of Tregs and enhances $T$ cell effector functions. To determine if AAVIL-27 treatment altered TME, we examined the cellular components of tumor-infiltrating leukocytes in B16 tumors from AAV-IL-27- or AAV-ctrl virus-treated mice using flow cytometry. As shown in Figure 2A, AAV-IL-27 treatment increased the percentage of CD45+ leukocytes in tumors. In the myeloid cell compart-

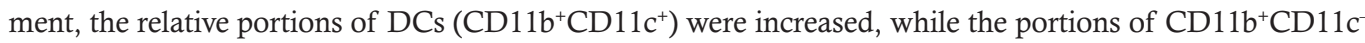
myeloid cells were reduced (Figure 2B). Moreover, we found that DC and myeloid cells in tumors from AAV-IL-27-treated mice had increased expression of MHC class II (Figure 2C). AAV-IL-27 treatment also enhanced tumor infiltration of NK cells (Figure 2D) and expression of Granzym B (Figure 2E) and Perforin (Figure 2F) in NK cells. Finally, we found that AAV-IL-27 treatment significantly reduced tumor infiltration of $\mathrm{CD} 19^{+} \mathrm{B}$ cells while it enhanced the infiltration of $\mathrm{CD}^{+} \mathrm{T}$ cells (Figure $2 \mathrm{G}$ ).

Since IL-27 is known to enhance Th1/Tc1 responses, we examined if $\mathrm{T}$ cell responses differed in B16 tumors from AAV-IL-27- and AAV-ctrl virus-treated mice. As shown in Figure 3A, we found that AAV-IL-27 treatment significantly increased the infiltration of both $\mathrm{CD}^{+}$and $\mathrm{CD} 8^{+} \mathrm{T}$ cells into 
A

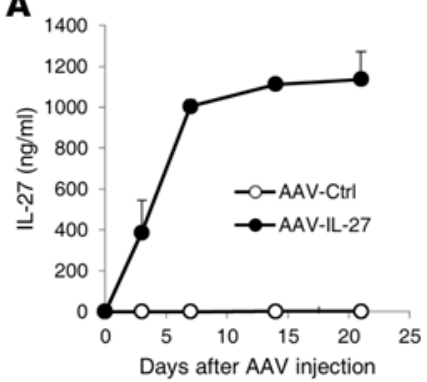

B

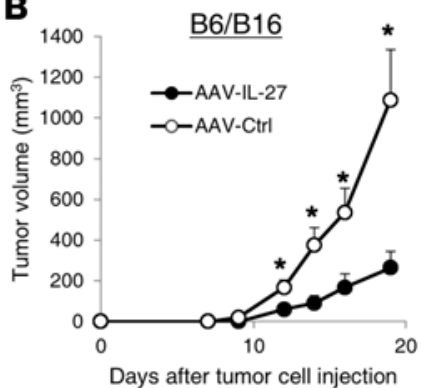

C

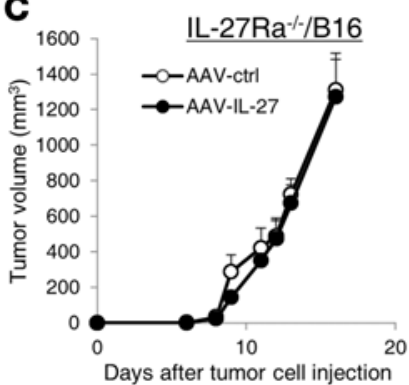

D

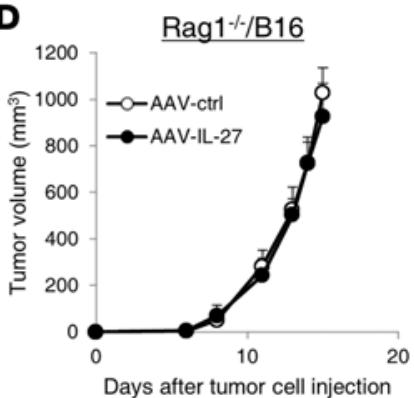

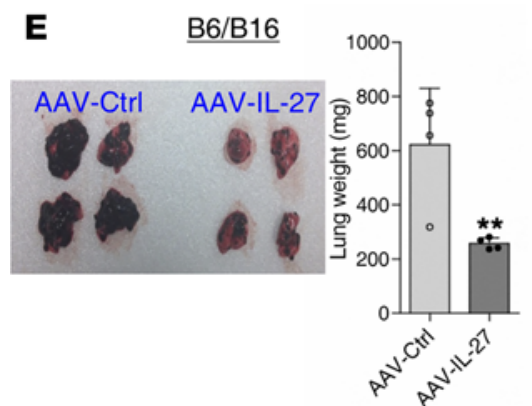
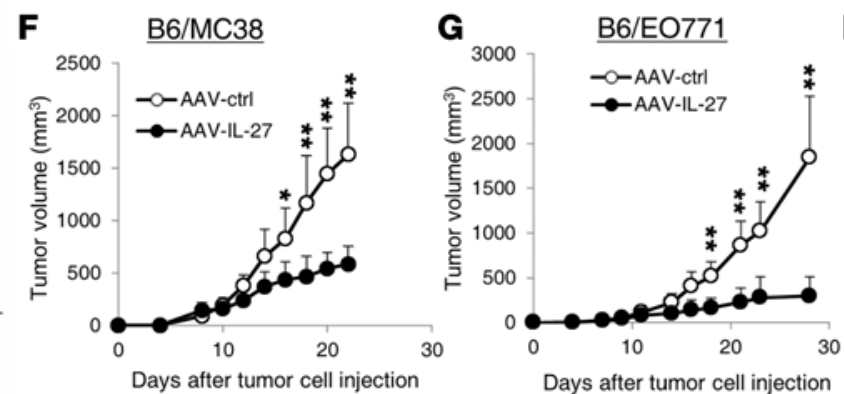

H

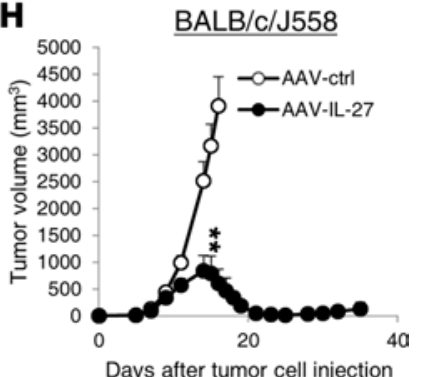

Figure 1. AAV-IL-27 treatment inhibits the growth and metastasis of tumors. (A) A single dose of AAV-IL-27 treatment resulted in sustained IL-27 production in mice. C57BL/6 mice were injected with AAV-IL-27 or AAV-ctrl viral vectors i.m. Mice were bled over time, and the concentrations of IL-27 in sera were detected by ELISA. Data represent mean \pm SD of 3-5 samples in each group/per time point. (B-D) AAV-IL-27 induced adaptive immunity to B16.F10 tumor. B16.F10 cells $\left(2 \times 10^{5}\right)$ were injected into C57BL/6 (B6/B16) (B), IL-27R $\alpha^{-1-}$ (C) and Rag1 ${ }^{-1-}$ mice (D) s.c. Four days later, mice were treated with AAV-IL-27 or AAV-ctrl viral vectors. Data represent mean \pm SD of 5 tumors in each group. Data shown represent 2-3 experiments with similar results. (E) AAV-IL-27 treatment inhibits melanoma lung metastasis. B16.F10 cells $\left(2 \times 10^{5}\right)$ were injected into C57BL/6 mice i.v. Four days later, mice were treated with AAV-IL-27 or AAV-ctrl viral vectors i.m. Twenty-one days after tumor cell injection, mice were sacrificed and tumor metastasis in the lungs were shown. Data in the right panel represent mean $\pm \mathrm{SD}$ of weights of the lungs from mice. Data shown represent 2 experiments with similar results. (F-H) Mice were injected with MC38 (F; $1 \times 10^{6}$ s.c.), E0771 (G; $1 \times 10^{6}$ intramammary), or $5558\left(\mathbf{H} ; 5 \times 10^{6}\right.$ s.c.) cells, followed by treatment with AAV-IL-27 or AAV-ctrl viral vectors 4 days later. Data are expressed as mean \pm SEM of 5 tumors in each group and represent 2 experiments with similar results. ${ }^{*} P<0.05,{ }^{* *} P<0.01$ by Student's $t$ test.

B16 tumors. The percentages of IFN- $\gamma$-producing $\mathrm{CD}^{+}$and $\mathrm{CD} 8^{+} \mathrm{T}$ cells in $\mathrm{B} 16$ tumors were also significantly increased (Figure 3B). Moreover, tumor-infiltrating CD8 ${ }^{+} \mathrm{T}$ cells from AAV-IL-27-treated mice also had increased expression of Granzyme B (Figure 3C) and Perforin (Figure 3D). Strikingly, we found that FoxP3 ${ }^{+}$Tregs were greatly reduced in spleens and B16 tumors of AAV-IL-27-treated mice (Figure 3E). Similarly, we found that mice with MC38 colon tumors treated with AAV-IL-27 also showed increased tumor infiltration of $\mathrm{CD}^{+}$and $\mathrm{CD} 8^{+} \mathrm{T}$ cells (Figure $3 \mathrm{~F}$ ), increased percentages of IFN- $\gamma$-producing $\mathrm{CD}^{+}$and $\mathrm{CD}^{+} \mathrm{T}$ cells (Figure $3 \mathrm{G}$ ), and greatly reduced Tregs in both spleen and tumors (Figure $3 \mathrm{H}$ ). Thus, AAV-IL-27 treatment enhances $\mathrm{T}$ cell effector functions in tumors and depletes Tregs in peripheral lymphoid organs and tumors.

AAV-IL-27 treatment inhibits IL-2R (CD25) expression in Tregs and IL-2 production by $T$ cells. We found that AAV-IL-27-mediated Treg depletion was a rapid process. By day 8 after AAV-IL-27 treatment, Tregs were already depleted in peripheral blood (Figure 4A). In spleens of AAV-IL-27-treated mice, we found that AAV-IL-27 treatment downregulated IL-2 receptor (CD25) expression, such that the CD $25^{+} \mathrm{FoxP}^{+}$Treg population was gradually reduced over time (Figure 4B). Since IL-2 is known to induce CD25 expression in T cells (27), and IL-27 is known to inhibit T cell secretion of IL-2 $(28,29)$, we examined if AAV-IL-27 treatment reduced IL-2 production by $\mathrm{T}$ cells. We found that, in AAV-IL-27-treated mice, IL-2 production by spleen $\mathrm{T}$ cells (Figure 4C) and tumor-infiltrating $\mathrm{T}$ cells (Figure 4D) was significantly reduced but was still maintained at significant levels, especially in spleens (Figure 4C). To test if heightened IL-2 signal prevents AAV-IL-27-mediated Treg depletion, we coinjected AAV-IL-2 with AAV-IL-27 and found that AAV-IL-2 treatment greatly enhanced the expression of CD25 in Tregs (Figure 4, E and F) and prevented their depletion in peripheral blood (Figure 4E) and spleen (Figure 4F). Thus, strengthening IL-2 signaling could upregulate CD25 expression in Tregs and prevent AAV-IL-27-mediated Treg depletion. 
A

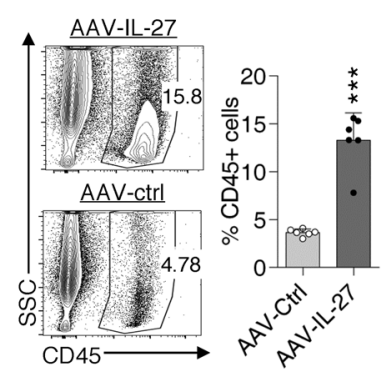

B

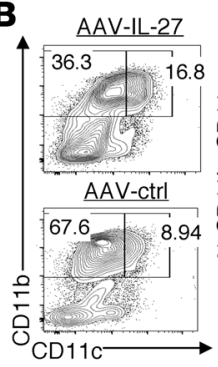

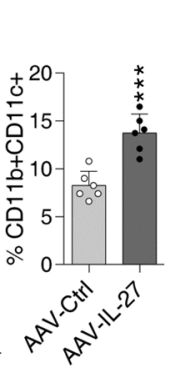
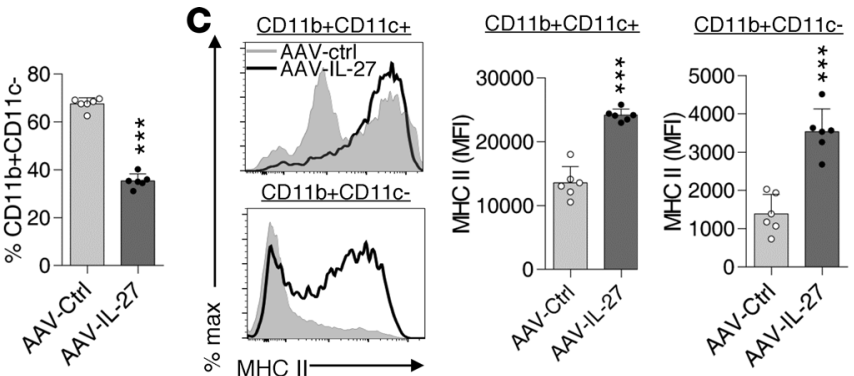

D

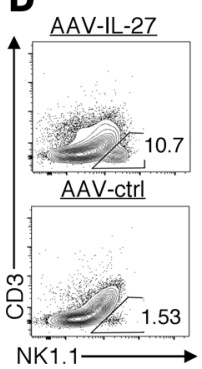

E

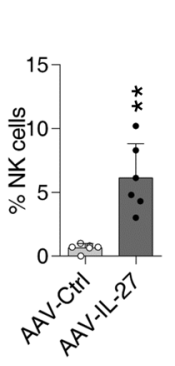

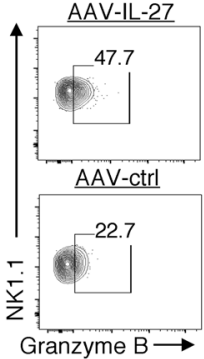

$\mathbf{F}$

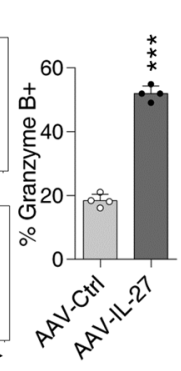

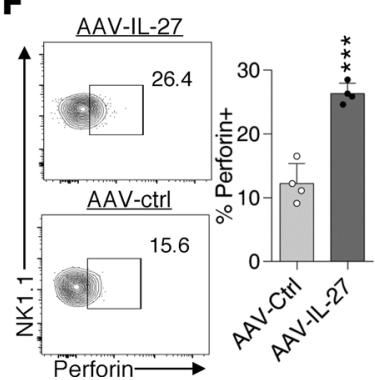

G

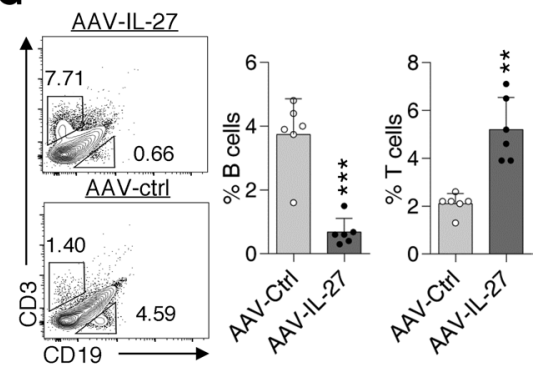

Figure 2. AAV-IL-27 therapy alters tumor microenvironment. B16.F10 cells $\left(2 \times 10^{5}\right)$ were injected into C57BL/6 mice s.C. Four days later, mice were treated with AAV-IL-27 or AAV-ctrl virus. Mice were sacrificed on day 21, and their tumors were analyzed for the infiltration of total leukocytes (A) and their subsets (B, D and $\mathbf{G})$. Expression of MHC class II on myeloid cells (C), Granzyme B (E), and Perforin (F) in NK cells were also quantified. Data were expressed as mean \pm SEM and represent $3-5$ experiments with similar results. ${ }^{*} P<0.01,{ }^{* *} P<0.001$ by Student's $t$ test.

AAV-IL-27-mediated Treg depletion is dependent on IL-27R-Stat1 signaling in Tregs. Unlike in WT mice, AAV-IL-27 treatment of IL-27R $\alpha^{-/-}$mice did not cause Treg reduction in spleens (Figure 5A) and tumors (Figure 5B). Similarly, AAV-IL-27 treatment of Stat $1^{-1-}$ mice also failed to deplete Tregs (Figure 5C). We further determined if the effect was Treg intrinsic or extrinsic using a mixed BM chimera approach. Briefly, BM cells from IL-27R $\alpha^{-/-}$mice $\left(\mathrm{CD} 45.2^{+}\right)$were mixed with $\mathrm{BM}$ cells from CD $45.1^{+} \mathrm{C} 57 \mathrm{BL} / 6$ mice at a 1:1 ratio and were injected into lethally irradiated $\mathrm{B} 6$ recipients to generate mixed $\mathrm{BM}$ chimeras. Twelve weeks after BM transfer, BM chimeric mice were injected with $0.2 \times 10^{6} \mathrm{~B} 16$. F10 cells per mouse s.c. and were treated with AAV-IL-27 or AAV-ctrl viruses 4 days later. As shown in Figure 5D, CD 45.2- vs CD $45.2^{+} \mathrm{CD} 4^{+} \mathrm{T}$ cells were largely at a $1: 1$ ratio in spleens of both groups, and AAV-IL-27

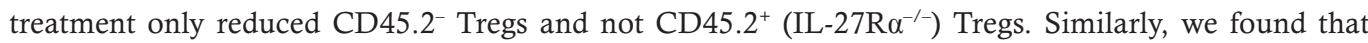
CD45.2- Tregs were nearly completely deleted in tumors (Figure 5E), while CD45.2+ Tregs were resistant to AAV-IL-27-mediated Treg deletion. These results together suggest that IL-27 signaling in Tregs mediates their depletion in AAV-IL-27-treated mice.

$A A V-I L-27$ treatment significantly enhances cancer vaccine efficacy. Tumor- (30) and vaccine-induced (31) expansion of Tregs is a significant obstacle to successful cancer immunotherapy. Since AAV-IL-27 treatment dramatically reduced Tregs in both peripheral lymphoid organs and tumors, we tested if AAV-IL-27 treatment could enhance cancer vaccine efficacy. We infected B16.F10 tumor cells with a lentivirus expressing GM-CSF or a control lentivirus, and we generated GM-CSF-secreting B16 tumor cells (B16-GMCSF) and control B16 cells (B16-ctrl). As expected, tumors established by B16-GM-CSF cells grew much slower compared with B16-ctrl cells (Figure 6A). However, we observed significant expansion of Tregs in mice with B16-GM-CSF tumors (Figure 6B). To determine if AAV-IL-27 and B16-GM-CSF vaccines had synergy in inhibiting tumor growth, C57BL/6 mice were immunized with irradiated B16-GM-CSF or B16-ctrl cells; 1 week later, the immunized mice were challenged with $2 \times 10^{5} /$ mouse of B16.F10 cells s.c. Four days after live tumor cell injection, mice were also treated with either AAV-IL-27 or AAV-ctrl vectors. We found that the combinations of AAV-IL-27/B16-ctrl- or B16-GM-CSF/AAV-ctrl-treated mice had significant reduced tumor growth (Figure 6C) compared with AAV-ctrl/B16-ctrl-treated mice. However, most significant tumor growth inhibition (Figure 6C) and prolonged survival (Figure 6D) were observed in AAV-IL-27/B16-GM-CSF combination-treated mice. As expected, Treg depletion was only observed in mice receiving AAV-IL-27 treatment (Figure 6E). 
A

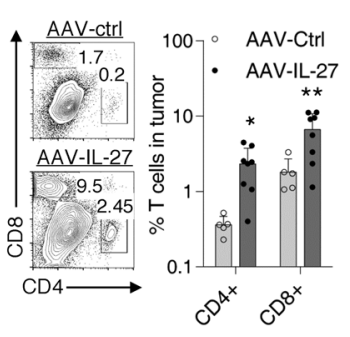

B

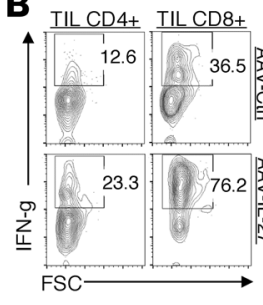

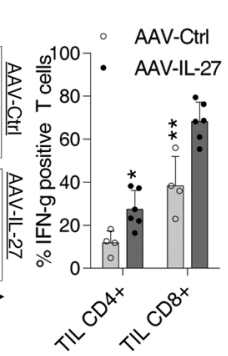

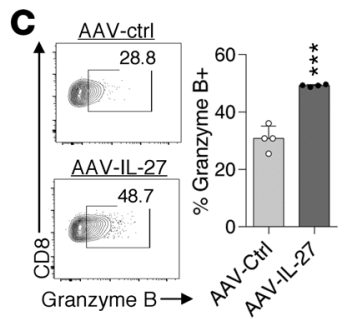

D

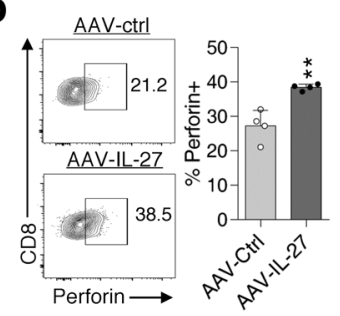

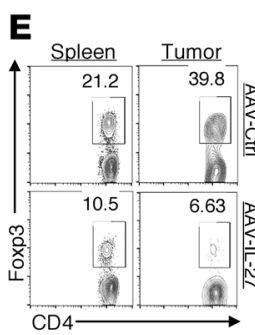

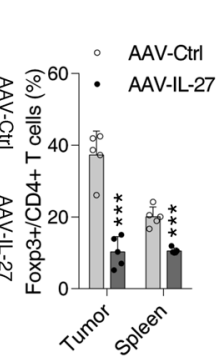

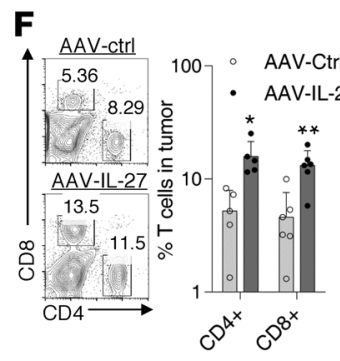

G
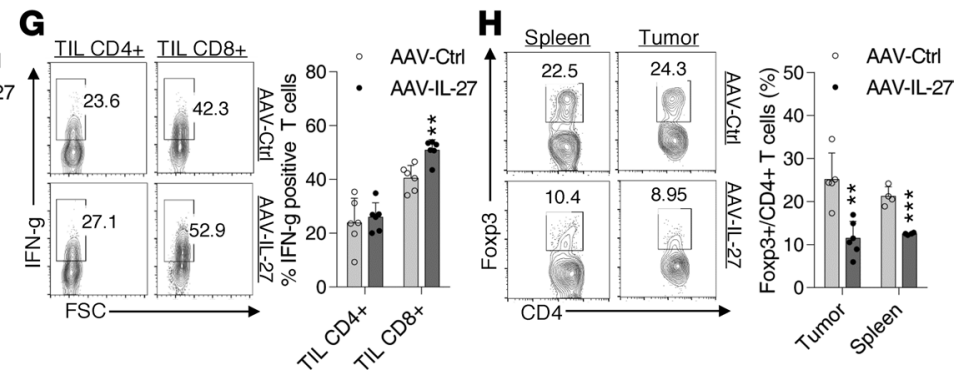

Figure 3. AAV-IL-27 treatment enhances T cell effector functions and depletes Tregs. B16.F10 cells $\left(2 \times 10^{5}\right)(\mathbf{A}-\mathbf{E})$ or $1 \times 10^{6}$ MC38 cells $(\mathbf{F}-\mathbf{H})$ were injected into C57BL/6 mice s.c. Four days later, mice were treated with AAV-IL-27 or AAV-ctrl virus. Mice were sacrificed on day 21, and their tumors were quantified for percentage of $\mathrm{CD}^{+}$and $\mathrm{CD} 8^{+} \mathrm{T}$ cells among total tumor mononuclear cells (A and $\mathbf{F}$ ), expression of IFN- $\gamma$ (B and $\left.\mathbf{C}\right)$, Granzyme B (C), and Perforin (D) in tumor-infiltrating T lymphocytes. Proportions of FoxP3 ${ }^{+}$Tregs $(\mathbf{E}$ and $\mathbf{H})$ in spleens and tumors from AAV-treated mice were also quantified. Data are expressed as mean \pm SEM and represent $2-5$ experiments with similar results. ${ }^{*} P<0.05$, ${ }^{* *} P<0.01,{ }^{* *} P<0.001$ by Student's $t$ test.

Recently, the whole genome/exosome sequencing of B16.F10 tumor cells has identified 21 neoantigens (32). We used IFN- $\gamma$ ELISPOT assay to evaluate $\mathrm{T}$ cell responses to those neoantigens to determine if AAV-IL-27/GM-CSF combination therapy induced more tumor antigen-specific T cells. As shown in Figure 6F, we found that spleens of AAV-IL-27/B16-GM-CSF-treated mice had globally enhanced T cell responses against those mutated peptides compared with AAV-ctrl/B16-GM-CSF-treated or other combination therapies. Thus, AAV-IL-27 treatment not only enhanced cancer vaccine efficacy, but also greatly boosted tumor antigen-specific $\mathrm{T}$ cell responses.

$A A V-I L-27$ therapy overcomes anti-PD-1 resistance. IL-27 was shown to induce the expression of programmed death-ligand 1 in $\mathrm{T}$ cells, which caused $\mathrm{T}$ cell tolerance via interaction with PD-1 on T cells (33). We found that $\mathrm{CD}^{+}$and $\mathrm{CD}^{+} \mathrm{T}$ cells from blood, spleen, and tumors receiving AAV-IL-27 treatment indeed upregulated PD-L1 expression (Figure 7A). Induction of PD-L1 expression was IL-27R dependent, since AAV-IL-27 treatment failed to induce T cell expression of PD-L1 in Il27ra ${ }^{-1-}$ mice (Figure 7B). Moreover, we found that AAV-IL-27-mediated induction of PD-L1 was compromised in Stat $1^{-/}$mice (Figure 7C), which is consistent with a critical role for Stat1 in IL-27 signaling (34).

To determine if IL-27-induced PD-L1 expression in T cells prevented the effectiveness of IL-27 therapy, C57BL/ 6 mice were injected with $2 \times 10^{5}$ B16.F10 cells s.c. and received the following treatments on day 4: AAV-ctrl virus + control mAb; AAV-ctrl virus + anti-PD-1; AAV-IL-27 virus + control mAb; or AAV-IL-27 virus + anti-PD-1. Antibodies were injected into mice at a dose of $300 \mu \mathrm{g} /$ mouse i.p. at 3-day intervals starting on day 4. As shown in Figure 7D, mice treated with AAV-IL-27 + control mAb showed significantly reduced tumor growth compared with mice treated with AAV-ctrl virus + control mAb or AAV-ctrl virus + anti-PD-1. Most significant inhibition of tumor growth was observed in mice treated with AAV-IL-27 + anti-PD-1 (Figure 7D). Overall, AAV-IL-27/anti-PD-1 combination therapy resulted in tumor-free survival in $50 \%$ of mice treated (Figure 7E). As expected, depletion of Tregs was only observed in groups of mice receiving AAV-IL-27 (Figure 7F). We used a similar regimen to treat mice with J558 tumors. As shown in Figure 7G, we found that J558 tumors grew progressively in mice treated with AAVctrl + ctrl Ab and AAV-ctrl + anti-PD-1; while in AAV-IL-27 + ctrl Ab-treated mice, tumors grew progressively, initially followed by significant regression. However, tumors eventually grew back in some mice from this group. In AAV-IL-27 + anti-PD-1-treated mice, tumors first grew into large masses, followed by complete regression (Figure 7G). Thus, in 2 tumor models that are resistant to anti-PD-1 therapy, we found that IL-27 gene therapy and anti-PD-1 combination could induce complete tumor rejection. 
A

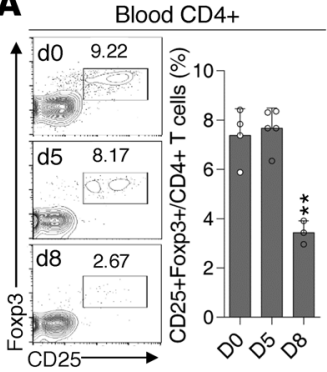

D Tumor

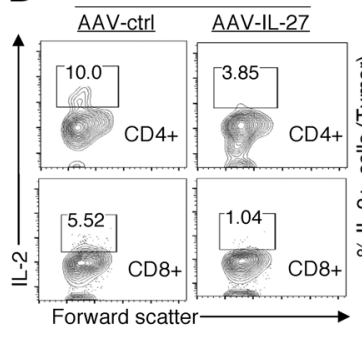

B

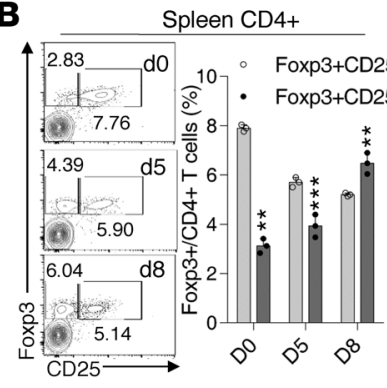

Spleen CD4+Foxp3+

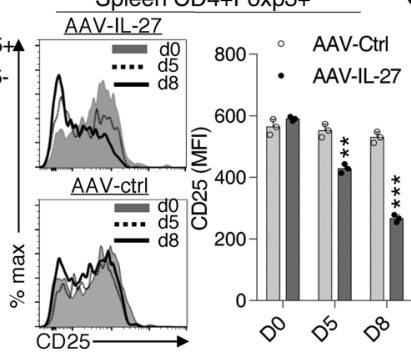

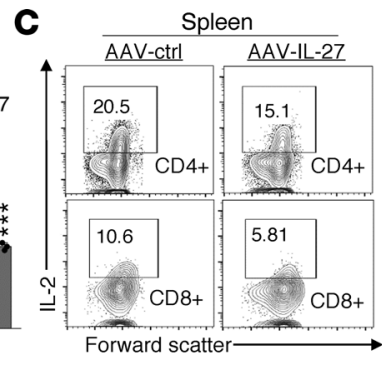

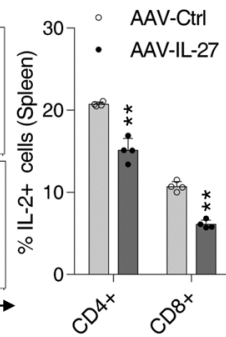

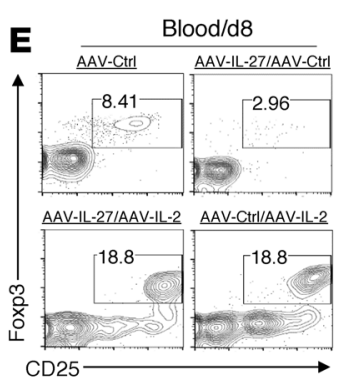
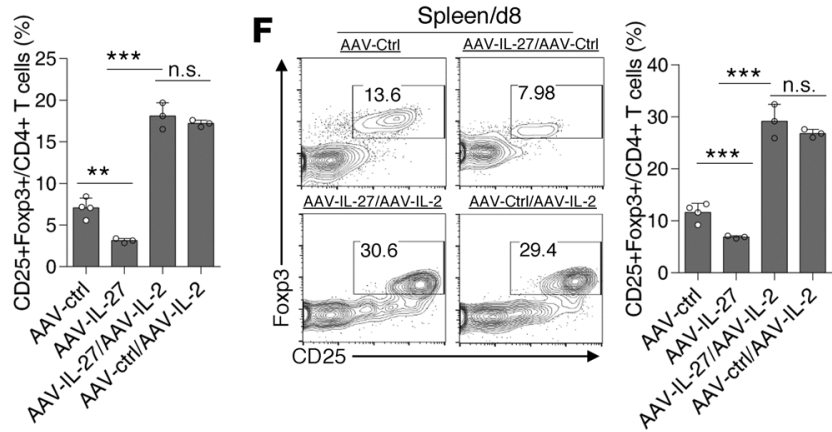

Figure 4. AAV-IL-27 inhibits IL-2 signaling. (A and B) C57BL6 mice were treated with AAV-IL-27 or AAV-ctrl virus i.m. at a dose of $2 \times 10^{11} \mathrm{DRP} / \mathrm{mouse}$. Treg numbers in blood (A) and spleens (B) were quantified at different times after AAV injection. Expression of CD25 in Tregs was also quantified over time (B, right panel). Data are expressed as mean \pm SEM and represent 2 experiments with similar results. ${ }^{* *} P<0.01,{ }^{* *} P<0.001$ by 1 -way ANOVA (A) or Student's $t$ test (B). (C and D) C57BL6 mice were injected with B16.F10 tumor cells $\left(2 \times 10^{5} /\right.$ mouse) s.c. Four days later, mice were treated with AAV-IL-27 or AAV-ctrl virus i.m. at a dose of $2 \times 10^{11} \mathrm{DRP} /$ mouse. Two weeks after viral injection, mice were sacrificed and T cells in spleens (C) and tumors (D) were analyzed for the expression of IL-2 by flow cytometry. Data are expressed as mean $\pm \mathrm{SEM}$ and represent 5 experiments with similar results. ${ }^{*} P<0.05,{ }^{* *} P$ $<0.01$ by Student's $t$ test. (E and $\mathbf{F}$ ) Cohorts of mice were treated with AAV-IL-27, AAV-IL-27/AAV-IL-2, AAV-ctrl/AAV-IL-2, or AAV-ctrl only. On day 8, Tregs in blood $(\mathbf{E})$ and spleens $(\mathbf{F})$ were analyzed and quantified by flow cytometry. Data are expressed as mean \pm SEM and represent 2 experiments with similar results. ${ }^{* *} P<0.01,{ }^{* *} P<0.001$ by 1 -way ANOVA test.

The role of IL-10 in AAV-IL-27-mediated antitumor activity and adverse events. IL-27 is known to induce IL-10 production by T cells (35); we have previously reported that IL-27 promotes T cells to produce IL-10 in vivo and that IL-10 production is critical for the antitumor activity of T cells in the J558 tumor model $(23,36)$. We first tested if AAV-IL-27 therapy induced IL-10 production in T cells in the treated mice. As shown in Figure 8A, AAV-IL-27 treatment induced significant numbers of IL- $10^{+} \mathrm{CD} 4^{+}$and $\mathrm{CD}^{+} \mathrm{T}$ cells in spleens. In $\mathrm{B} 16$ tumors, AAV-IL-27 treatment resulted in the depletion of IL-10-producing Tregs, while it induced IL-10 production in effector $\mathrm{CD}^{+}$and $\mathrm{CD} 8^{+} \mathrm{T}$ cells (Figure 8B). In the $\mathrm{B} 16$ tumor model (Figure 8C), we found that AAV-IL-27 treatment significantly inhibited tumor growth in both $\mathrm{IL}-10^{-/-}$and WT mice, but with a slightly increased efficacy in IL-10-- mice compared with its action in WT mice. In the plasmacytoma J558 tumor model, while WT mice were highly susceptible to AAV-IL-27 therapy, we found that IL-10-/ mice were less responsive to AAV-IL-27 therapy (Figure $8 \mathrm{D}$ ); as a result, the majority of mice eventually succumbed to tumor growth (Figure 8E). Thus, IL-10 was required for AAV-IL-27-mediated antitumor activity in the J558 tumor model and was not required in the B16 model.

Systemic depletion of Tregs raised a concern of developing autoimmune diseases in mice treated with AAV-IL-27. This concern is especially valid since IL-27 transgenic mice have been shown to develop Scurfylike diseases (37). However, we did not find any symptoms of autoimmune diseases in AAV-IL-27-treated $\mathrm{BALB} / \mathrm{c}$ mice that rejected J558 tumors. We further examined if mice developed pathology at a histological level in AAV-IL-27-treated WT and IL-10/- mice. As shown in Figure 9A, at 2 months after treatment, we did not find inflammatory lesions in the heart, lung, liver, pancreas, kidney, or colon in AAV-IL-27treated WT mice. However, in AAV-IL-27-treated IL-10-deficient mice, we found perivascular inflammatory lesions in the liver but not in other organs (Figure 9A). To further understand if perivascular inflammatory lesions in the liver are IL-27 therapy specific, we treated more WT and IL- $10^{-/-} \mathrm{BALB} / \mathrm{c}$ mice with AAV-IL-27 or AAV-ctrl virus, and we found that only AAV-IL-27-treated IL-10-/- mice developed liver inflammatory lesions 2 months later (Figure 9, B and C). Thus, IL-10 appears to be a significant contributor to the protection of liver inflammation in AAV-IL-27-treated mice. 
A

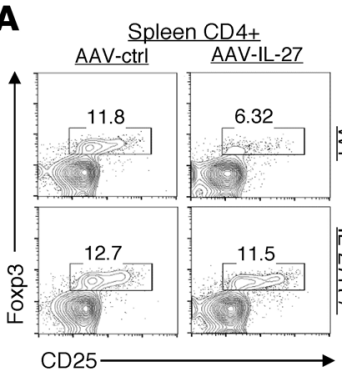

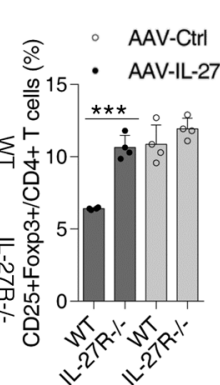

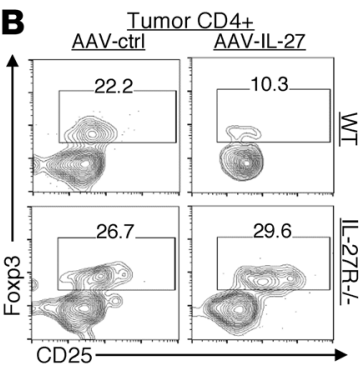

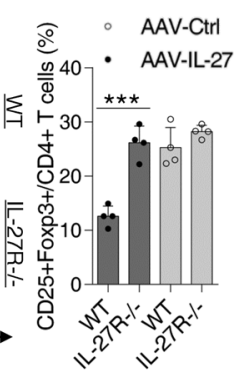
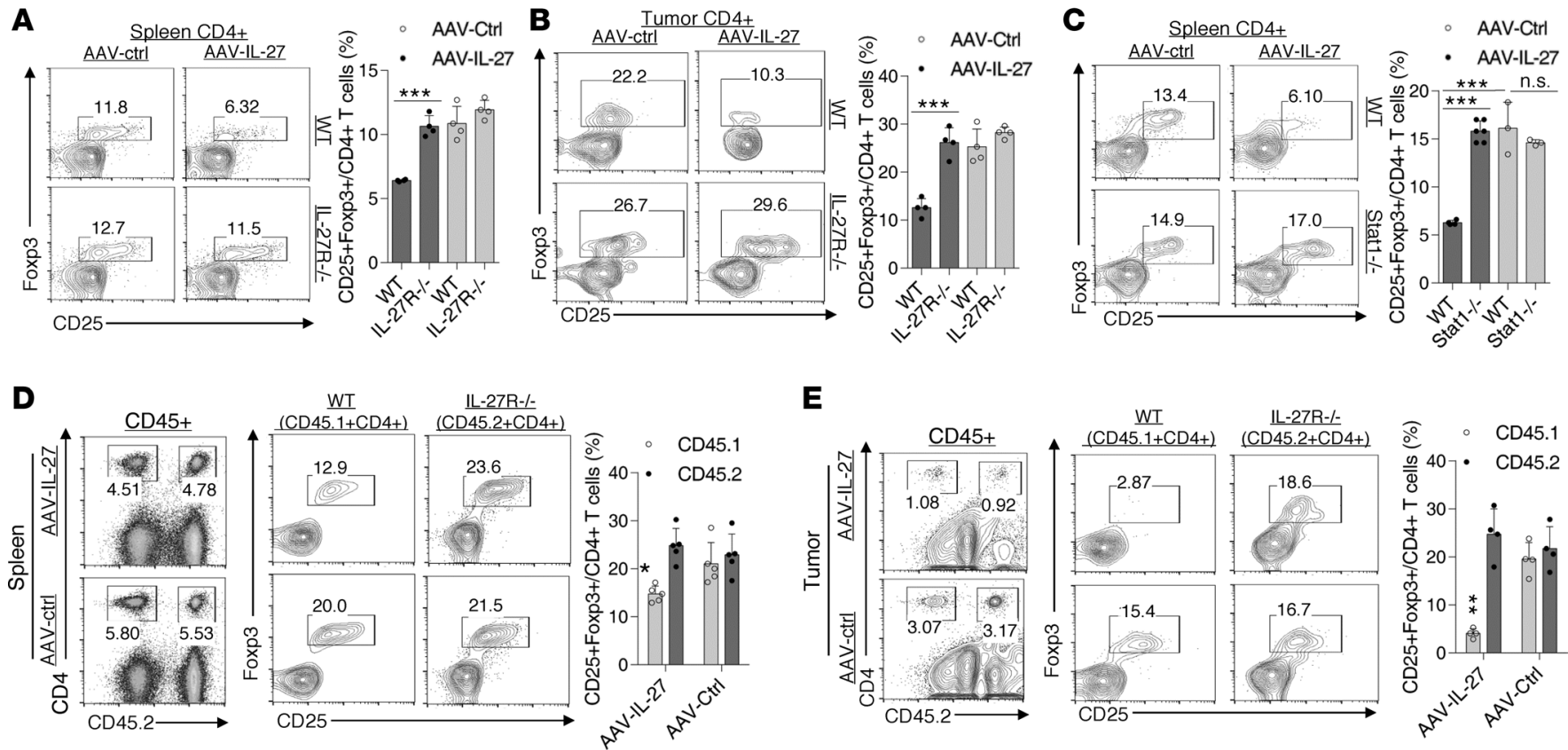

Figure 5. IL-27 signaling in Tregs mediates Treg depletion. (A and B) IL-27ra ${ }^{-/-}$and C57BL6 mice were injected with B16.F10 tumor cells ( $\left.2 \times 10^{5} / \mathrm{mouse}\right) \mathrm{s} . \mathrm{c}$. Four days later, mice were treated with AAV-IL-27 or AAV-ctrl virus i.m. at a dose of $2 \times 10^{11} \mathrm{DRP} /$ mouse. Fourteen days after viral injection, mice were sacrificed and their spleens (A) and tumors (B) were analyzed for the presence of Tregs by flow cytometry. Data are expressed as mean \pm SEM and represent 2 experiments with similar results. ${ }^{* *} P<0.001$ by Student's $t$ test. (C) Stat1 ${ }^{-1-}$ and BALB/C mice were treated with AAV-IL-27 or AAV-ctrl virus i.m. at a dose of $2 \times 10^{11} \mathrm{DRP} / \mathrm{mouse}$. Fourteen days after treatment, mice were sacrificed, and their spleens were analyzed for the presence of Tregs by flow cytometry. Data are expressed as mean \pm SEM and represent 2 experiments with similar results. ${ }^{* *} P<0.001$ by Student's $t$ test. ( $D$ and $\mathbf{E}$ ) Mixed BM chimeric mice (CD45.2 $2^{+} \mathrm{IL}-27 \mathrm{R} \alpha^{-1-} / \mathrm{CD} 45.1^{+}$C57BL/6) were injected with B16.F10 tumor cells and treated with AAV-IL-27 or AAV-ctrl vectors 4 days later. Twenty-one days after tumor cell injection, mice were sacrificed, CD45 ${ }^{+}$spleen cells (D) and tumor-infiltrating leukocytes (E) were analyzed for CD4/CD45.2 (left panels) and CD4/CD25/FoxP3 (right panels) by flow cytometry. Data are expressed as mean \pm SEM and represent 2 experiments with similar results. ${ }^{*} P<0.05,{ }^{* *} P<0.01$ by Student's $t$ test.

\section{Discussion}

In this study, we have developed an innovative and effective gene therapy (i.e., using AAV-IL-27 to treat mice with tumors). We found that monotherapy with AAV-IL-27 significantly inhibited the growth of a broad spectrum of cancer types in experimental mouse models. In combination with a GM-CSF vaccine, AAV-IL-27 treatment induced nearly complete tumor inhibition and long-term survival of mice. Moreover, we found that AAV-IL-27 also dramatically increased the efficacy of anti-PD-1 therapy.

A number of indications suggest that AAV-IL-27-induced $\mathrm{T}$ cell response plays key role in its antitumor efficacy. First, we found that AAV-IL-27 therapy only showed efficacy in immune-competent mice but not in Rag1-deficient mice lacking $\mathrm{T}$ and B lymphocytes. Second, through the analysis of tumor-infiltrating leukocytes, we found that AAV-IL-27 therapy enhanced T cell, but not B cell, infiltration into tumors, and tumor-infiltrating $\mathrm{CD}^{+} \mathrm{T}$ cells and conventional $\mathrm{CD} 4^{+} \mathrm{T}$ cells had increased effector functions. Third, we found that AAV-IL-27 therapy also increased DC and NK cell infiltration into tumors. Tumor-infiltrating DCs upregulated MHC class II, and NK cells expressed more Perforin and Granzyme B, suggesting that NK-mediated killing of tumor cells and DC-mediated cross-priming of $\mathrm{T}$ cells play important roles in AAV-IL-27-mediated T cell response. Fourth, in mice treated with AAV-IL-27 and GM-CSF vaccine, neoantigen-specific $\mathrm{T}$ cell responses were greatly enhanced. Fifth and most strikingly, we found that AAVIL-27 treatment had a potent effect on depletion of Tregs in peripheral lymphoid organs and, more pronouncedly, in tumors, which could further boost $\mathrm{T}$ cell responses. While the above observations are consistent with some of the known functions of IL-27 in tumor immunity (20-22), to our knowledge, this study is the first demonstration that therapeutic application of IL-27 causes systemic Treg depletion, which has a significant impact on antitumor $\mathrm{T}$ cell responses and cancer immunotherapy.

A previous study (37) has revealed that, in IL-27 transgenic mice, Tregs were found to be deleted, and inhibition of IL-2 production by T cells was considered to be the mechanism responsible for Treg depletion in IL-27 transgenic mice (37). However, in this study, we found that reduction of IL-2 does not fully explain AAV- 
A 3500

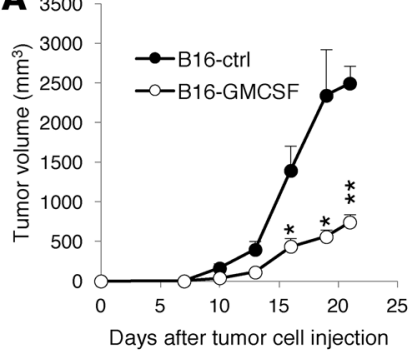

B

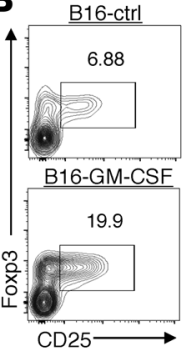

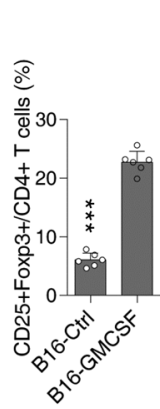

$\mathbf{C}_{3000}$

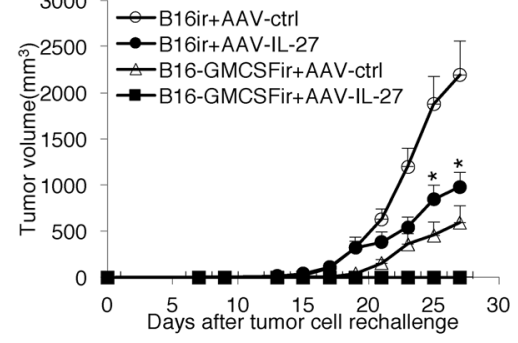

D

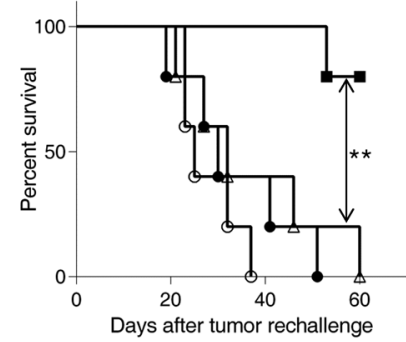

E

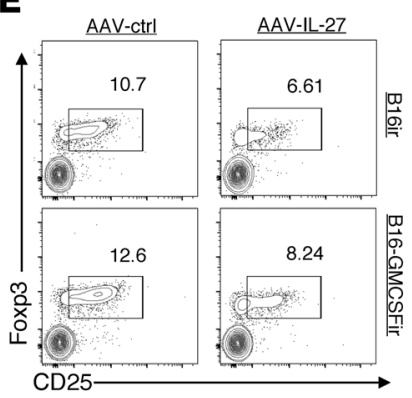

$\mathbf{F}_{250}$

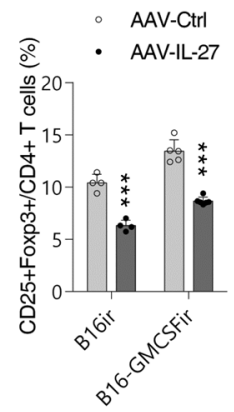

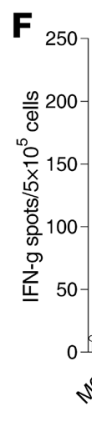

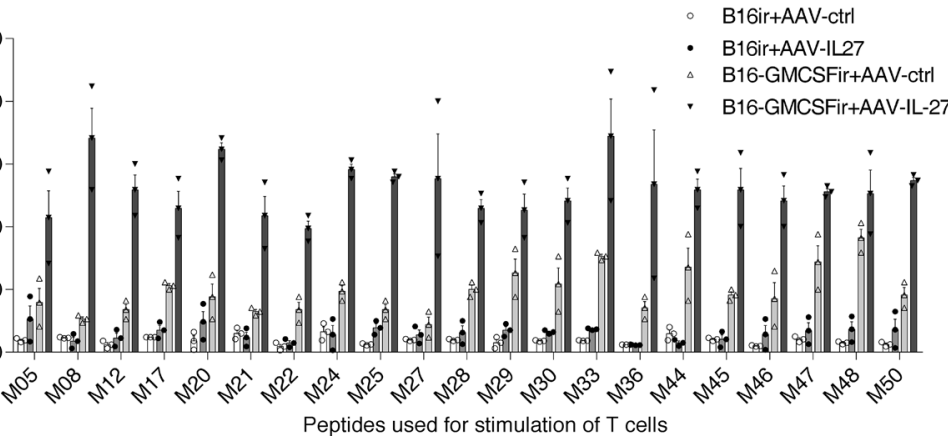

Figure 6. AAV-IL-27 treatment enhances cancer vaccine efficacy and boosts antitumor T cell responses. (A and B) C57BL6 mice were injected with B16GM-CSF $(n=5)$ or B16-ctrl cells $(n=5)$ at a dose of $0.2 \times 10^{6} /$ mouse. Mice were observed for tumor growth over time (A). By day 21 , mice were sacrificed and $\mathrm{CD}^{+} 5^{+} \mathrm{FoxP3}^{+}$Tregs in spleens were quantified by flow cytometry (B). Data are expressed as mean $\pm \mathrm{SEM}$ and represent 2 experiments with similar results. ${ }^{*} P<0.05,{ }^{* *} P<0.01$, ${ }^{* *} P<0.001$ by Student's $t$ test. (C-E) C57BL6 mice were immunized with irradiated B16-GM-CSF $(n=5)$ or B16-ctrl cells $(n=5)$ at a dose of $0.2 \times 10^{6} /$ mouse. One week later, mice were challenged with B16.F10 cells ( $0.2 \times 10^{6} /$ mouse) s.c. On day 4 , after tumor cell challenge, AAV-IL-27 or AAV-ctrl virus were injected i.m. at a dose of $2 \times 10^{11} \mathrm{DRP} /$ mouse. Tumor volume changes (C), mice survival (D), and Treg numbers in spleens (E) were examined. Data are expressed as mean \pm SEM and represent 3 experiments with similar results. ${ }^{*} P<0.05,{ }^{* *} P<0.01$, ${ }^{* * *} P<0.001$ by Student's $t$ test $(\mathbf{A}$, B, E), 1-way ANOVA (C), or log-rank test (D). (F) IFN- $\gamma$ ELISPOT assay was used to evaluate T cell responses to peptide neoantigens in B16.F10 cells (peptide sequences published in re. 32). Splenic T cells were pooled from 3 mice in each group. Data are expressed as mean \pm SEM of counts in 3 replicate wells and represent 3 experiments with similar results. IFN- $\gamma$ spot numbers in splenic T cells were significantly increased in AAV-IL-27/B16-GM-CSF-treated mice compared with AAV-ctrl/B16-GM-CSF-treated mice ( $P<0.05$ by 1-way ANOVA) under all peptide conditions.

IL-27-mediated Treg depletion, since we found that AAV-IL-27-mediated inhibition of IL-2 production was not dramatic, especially in peripheral lymphoid organs (Figure 4). Experiments involving mixed BM chimera and gene-deficient mice clearly suggested that AAV-IL-27-mediated Treg depletion depended on IL-27R and Stat1 signaling in Tregs (Figure 5). Since AAV-IL-27 therapy downregulates CD25 expression in Tregs (Figure 4), we consider that inhibition of IL-2 signaling in Tregs by IL-27 is the major mechanism for AAV-IL-27-mediated Treg depletion. Mechanistically, IL-27-mediated activation of Stat1 is known to induce the expression of Socs3 (29); Socs3 can inhibit IL-2-induced activation of Jak1 (38), leading to compromised Stat5 activation and subsequent CD25 expression (39). Indeed, expression of Socs 3 in Tregs has been shown to inhibit Treg proliferation, survival, and effector functions (40). Heightened IL-2 signaling can prevent AAV-IL-27-mediated Treg depletion, suggesting that this mechanism is active when IL-2 signaling is low. More complete inhibition of IL-2 in tumors explains why depletion of Tregs is more complete in TME (Figure 4).

Tumor-induced expansion of Tregs is a significant obstacle to successful cancer immunotherapy (30). Vaccines can prime $\mathrm{T}$ cell responses to tumor antigens. However, vaccination is usually accompanied by the expansion of Tregs (31), which can limit the expansion of tumor antigen-specific T cells. GM-CSF-secreting tumor vaccine is a potent inducer of antitumor $\mathrm{T}$ cell responses (41). In this study, we found that it had a similar efficacy of AAV-IL-27 monotherapy. However, these 2 therapeutic together have potent synergy in inducing antitumor T cell responses to neoantigens in B16 tumors, leading to long-term survival of mice. These data suggest that the Treg-depletion ability of AAV-IL-27 and the ability of T cell priming by GMCSF vaccine are probably an ideal combination for cancer immunotherapy.

Another significant observation in this study is that AAV-IL-27 enhances cancer sensitivity to antiPD-1 therapy (Figure 7). The dramatic synergy of the AAV-IL-27 and anti-PD-1 therapy can be explained based on the following observations. First, AAV-IL-27 induces T cell infiltration into tumors (Figure 2 
A

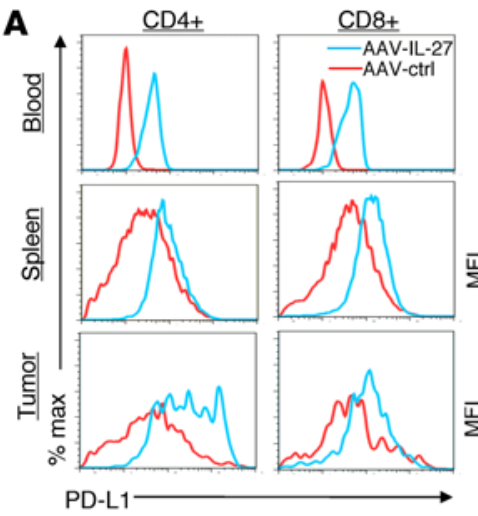

E $\underline{B 16}$

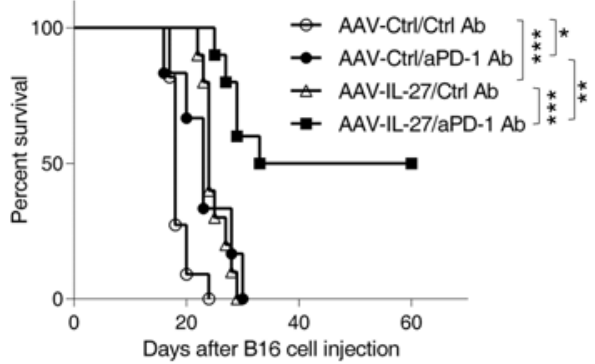

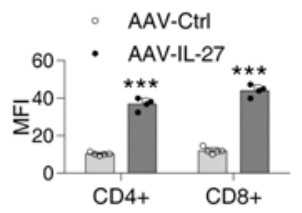
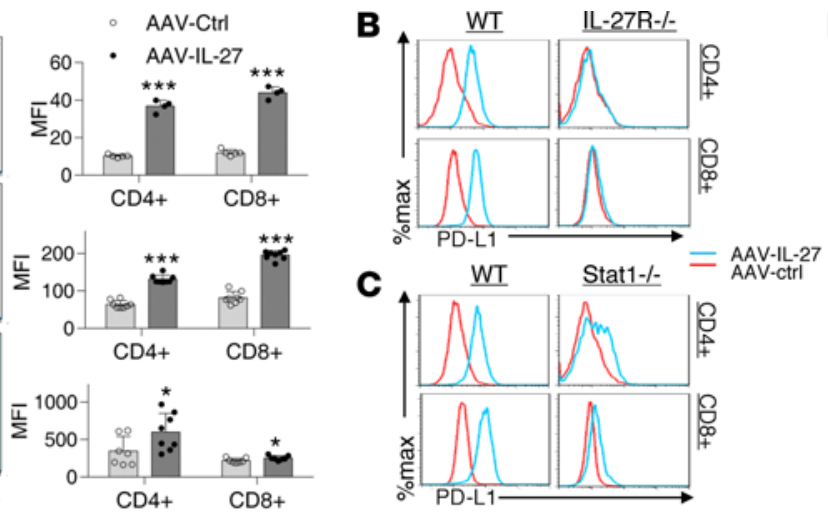

F Tumor $\mathrm{CD} 4+$
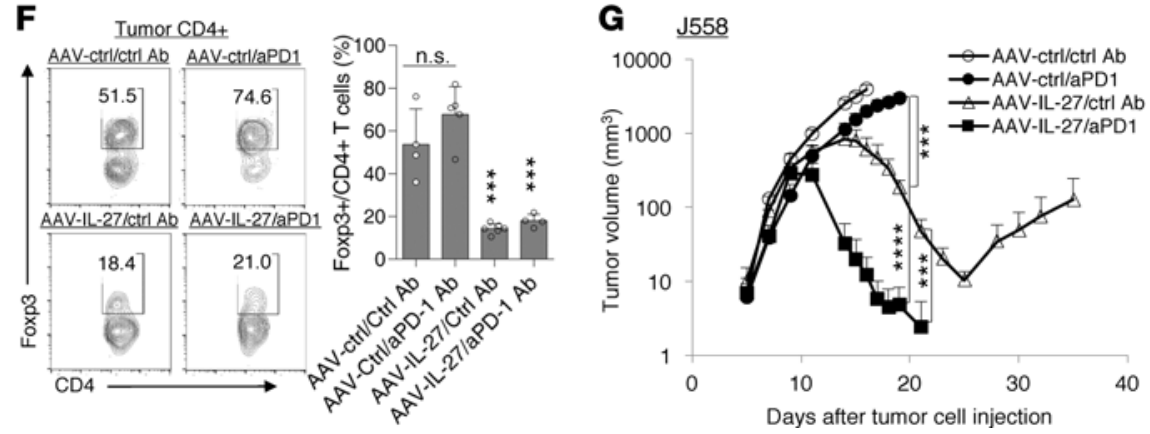

Figure 7. AAV-IL-27 treatment induces PD-L1 expression in T cells and enhances tumor sensitivity to anti-PD-1 therapy. (A) Flow cytometry analysis of $\mathrm{CD}^{+}$and $\mathrm{CD} 8^{+} \mathrm{T}$ cells in the blood, spleens, and tumors from mice that were treated with AAV-IL-27 or AAV-ctrl viral vectors. Average mean fluorescence intensity (MFI) of PD-L1 expression in T cells from each group of mice was shown in the right panel. Data are expressed as mean \pm SEM and represent 5 experiments with similar results. ${ }^{*} P<0.05,{ }^{* *} P<0.001$ by Student's $t$ test. (B and $\mathbf{C}$ ) Flow cytometry analysis of PD-L1 expression in spleen T cells from IL-27ra ${ }^{-/-}$(B) and Stat1 ${ }^{-/-}$(C) mice that were treated with AAV-IL-27 or AAV-ctrl viral vectors. Data shown represent 2 experiments involving at least 5 mice with similar results. (D-F) AAV-IL-27 and anti-PD-1 therapies show synergy in mice with B16.F10 melanoma. B16.F10 cells $\left(2 \times 10^{5}\right)$ were injected into each C57BL6 mice s.c. Four days later, mice were treated with AAV-IL-27 or AAV-ctrl vectors i.m. Starting on day 4, mice were also treated with $300 \mu \mathrm{g} /$ mouse of anti-PD-1 (RMP1-14) or an isotype-matched control antibody (2A3) at 3-day intervals for up to 4 times. Tumor volume changes (D, $n=5$ /group), mice survival (E, $n=8-11$ /group), and Treg numbers in tumors (F) were examined. Data are expressed as mean \pm SEM and represent 3 experiments with similar results. ${ }^{*} P<0.05,{ }^{*} P<0.01,{ }^{* *} P<0.001$ by 1 -way ANOVA (D, F, G) or log-rank test (E). (G) Synergy of anti-PD-1 and AAVIL-27 in treating mice with plasmocytoma 558 tumors. $J 558$ cells $\left(5 \times 10^{6}\right)$ were injected into each BALB/c mouse s.c. Four days later, mice were treated with AAV-IL-27 or AAV-ctrl vectors i.m. Starting on day 4, mice were also treated with $300 \mu \mathrm{g} / \mathrm{mouse}$ of anti-PD-1 (RMP1-14) or an isotype-matched control antibody $(2 \mathrm{~A} 3)$ at 3 -day intervals for up to 4 times. Five mice per group were used for this study. Data are expressed as mean $\pm \mathrm{SEM}$ and represent 3 experiments with similar results. ${ }^{* *} P<0.001$ by 1 -way ANOVA.

and Figure 3), which is considered to be a prerequisite for PD-1 blockade therapy to work (42). Second, AAV-IL-27 induces PD-L1 by a largely Stat1-dependent mechanism (Figure 7, A-C). Since PD-L1-PD-1 interaction is known to limit T cell effector functions $(33,42)$, PD-1 blockade can remove the limitation of AAV-IL-27-mediated PD-L1 induction in T cells, leading to rejuvenated T cell effector functions and the control of tumor growth. Interestingly, a recent study suggests that PD-L1 expression in host immune cells is more related to cancer sensitivity to anti-PD-L1 therapy (43). Thus, our results suggest that induction of PD-L1 in T cells by IL-27 contributes to tumor sensitivity to anti-PD-1 therapy. Third, AAV-IL-27 reduces Tregs in both TME and lymphoid organs in an IL-27R- and Stat1-dependent manner. Tregs in the TME have been shown to contribute to anti-PD-1 resistance (44). Thus, our data provide a compelling rationale for combination therapy using AAV-IL-27 and anti-PD-1.

Current strategies to deplete Tregs to promote antitumor immunity have been met with considerably challenges. On one hand, IL-2 and diphtheria toxin fusion protein could target $\mathrm{CD} 25^{+} \mathrm{T}$ cells, but they were often found to be inefficient in depleting Tregs in a human trial (45). On the other hand, experimental studies in mice have shown effective depletion of Tregs in adult mice but lead to severe and even lethal autoimmune diseases (46). Our data presented herein offers a new approach that can effectively deplete Tregs without causing signifcant adverse events. We consider the following mechanisms operative in preventing autoimmunity in AAV-IL27-treated mice. First, AAV-IL-27-mediated Treg depletion is incomplete in lymphoid organs, and the residual Tregs may be sufficient to prevent the development of autoimmunity. Second, AAV-IL-27 can directly inhibit 
A
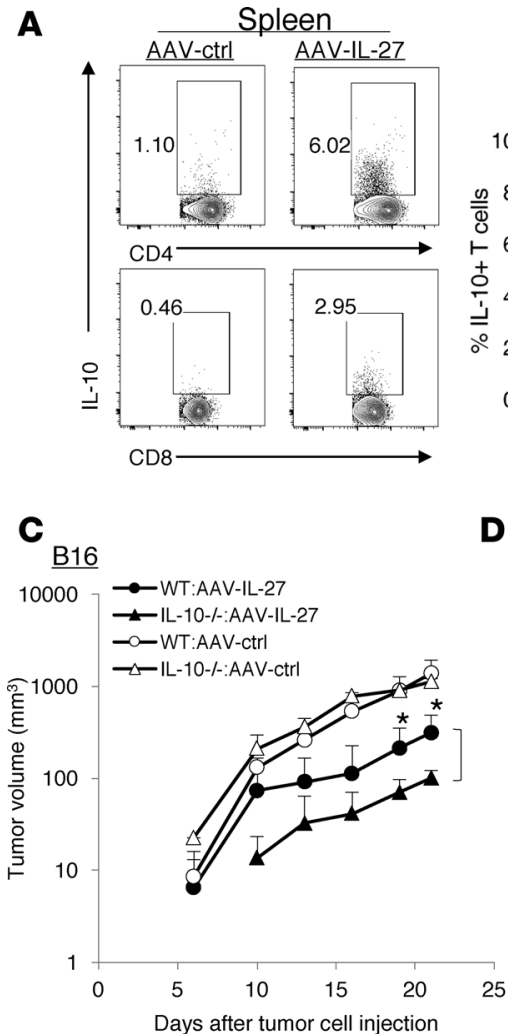

D
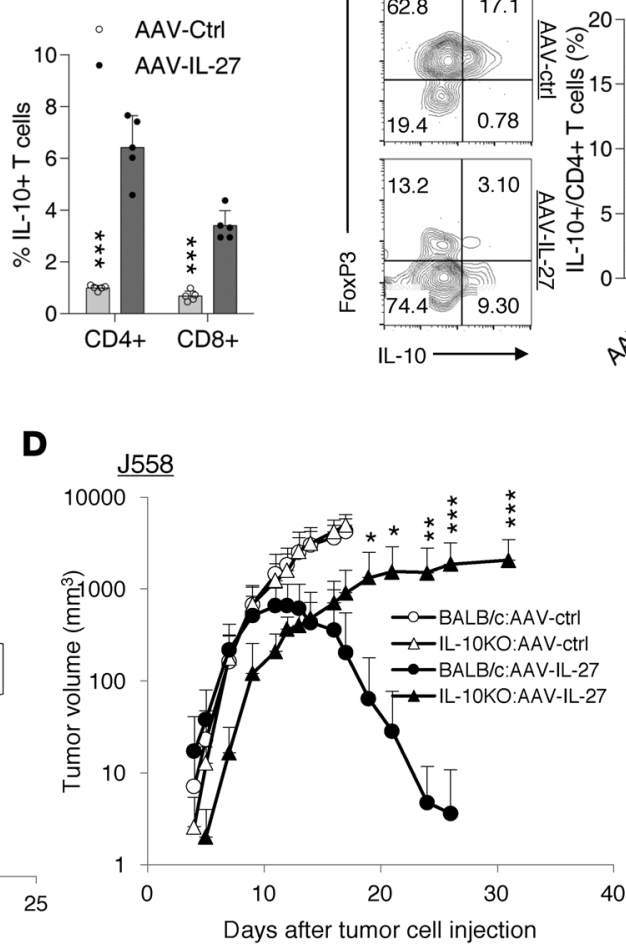

B

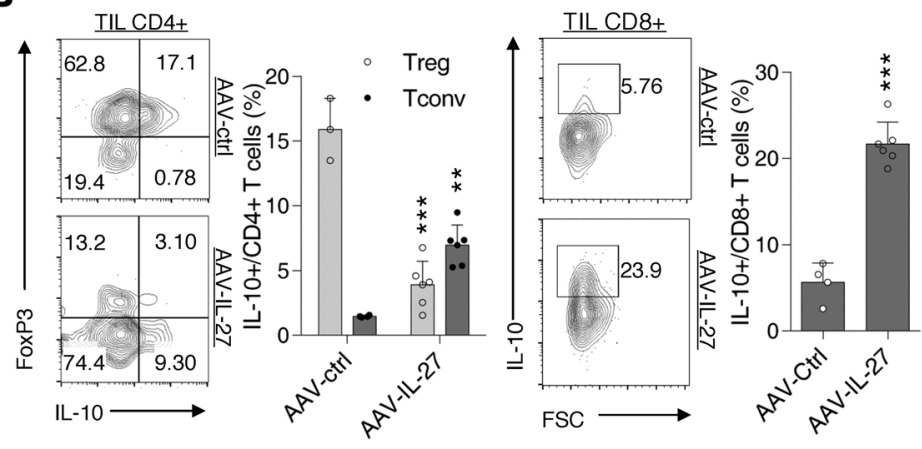

$\mathbf{E}$

J558

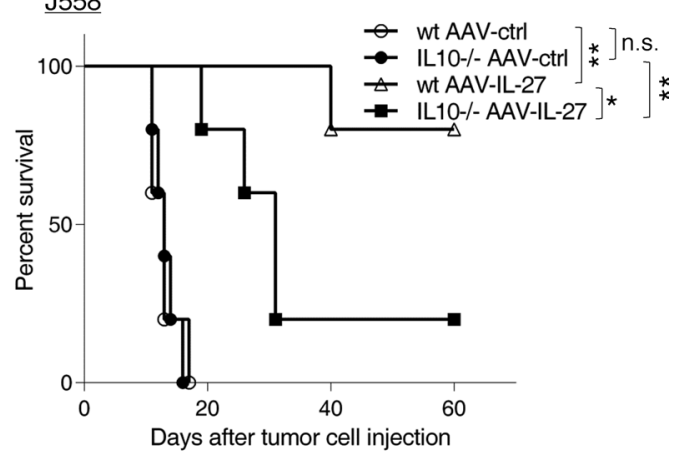

Figure 8. The role of IL-10 in AAV-IL-27-mediated antitumor activity. (A and B) B16.F10 cells $\left(2 \times 10^{5}\right)$ were injected into C57BL/6 mice s.c. Four days later, mice were treated with AAV-IL-27 or AAV-ctrl virus i.m. at a dose of $2 \times 10^{11} \mathrm{DRP} /$ mouse. Percentages of IL-10+ $\mathrm{CD} 4^{+}$and $\mathrm{CD} 8^{+} \mathrm{T}$ cells in spleen (A) and tumors (B) were quantified by flow cytometry. Data are expressed as mean $\pm \mathrm{SEM}$ and represent at least 5 experiments with similar results. ${ }^{* *} P<0.01,{ }^{* * *} P<0.001$ by Student's $t$ test. (C) B16.F10 cells $\left(2 \times 10^{5}\right)$ were injected into C57BL/ 6 and IL10-I-C57BL6 mice s.c. Four days later, mice ( $n=5 /$ group) were treated with AAVIL-27 or AAV-ctrl virus i.m. at a dose of $2 \times 10^{11} \mathrm{DRP} /$ mouse. Data are expressed as mean $\pm \mathrm{SEM}$ and represent 2 experiments with similar results. ${ }^{*} P<0.05$ by one-way ANOVA test. ( $\mathbf{D}$ and $\mathbf{E}) \mathrm{J} 558$ cells $\left(5 \times 10^{6}\right)$ were injected into BALB/c and IL10-I-BALB/c mice s.c. Four days later, mice $(n=5 / \mathrm{group})$ were treated with AAV-IL-27 or AAV-ctrl virus i.m. at a dose of $2 \times 10^{11} \mathrm{DRP} /$ mouse. Tumor volumes (D) and mice survival (E) were observed. Data are expressed as mean $\pm \mathrm{SEM}$ and represent 2 experiments with similar results. ${ }^{*} P<0.05,{ }^{* *} P<0.01,{ }^{* * *} P<0.001$ by 1 -way ANOVA $(\mathbf{C}$ and $\mathbf{D})$ or log-rank test (E).

autoimmune Th2 and Th17 responses via blocking the expression of transcription factors GATA-3 (Th2) and $\operatorname{RoR} \gamma \tau$ (Th17) $(16,17)$ and can induce a number of inhibitory pathways in T cells $(47)$, which may potentially prevent the development of autoimmunity. We recently showed that AAV-IL-27 therapy could block the development of autoimmune colitis by induction of PD-L1 (48). Third and most likely, AAV-IL-27-induced IL-10producing $\mathrm{T}$ cells play a role in preventing adverse events. IL-10 is a major cytokine that defines a major subset of Tregs and plays a critical role in Treg function. By promoting IL-10 production in effector T cells, AAV-IL-27 therapy may have bypassed the need for Tregs in protection against autoimmune adverse events. Although, at this stage, we are not sure if the observed liver inflammation in AAV-IL-27-treated IL-10-/- mice (Figure 9) was due to autoimmunity or direct toxicity of excessive IL-27, the fact that the liver inflammation was not observed in WT mice suggests a key role for IL-10 in preventing adverse events related to AAV-IL-27 therapy. In addition to preventing liver inflammation, our data (Figure 8) also suggest that AAV-IL-27-induced IL-10 may contribute to antitumor activity in at least some tumor models. This observation is consistent with the promising clinical trial results using recombinant IL-10 to treat cancer patients (49). Thus, AAV-IL-27-induced IL-10 could play dual roles in cancer immunotherapy: preventing adverse events and promoting antitumor immunity.

While some studies $(37,50)$, including the current study, report a role of IL-27 in limiting Treg population, there are other studies (51-53) that suggest that IL-27 does not limit Treg populations but enhances the inhibitory functions of Tregs. These seemingly controversial results reflect the pleiotropic roles of IL-27 and could be explained by IL-27 dose and the presence of IL-2 signaling in experimental systems. Treg depletion occurs in IL-27 transgenic mice and mice receiving AAV-IL-27 therapy, suggesting that depletion of Tregs by IL-27 probably requires higher doses of IL-27, which is not present under physiological conditions and the majority of pathological conditions. Additionally, IL-2 was used in Treg cultures in in 

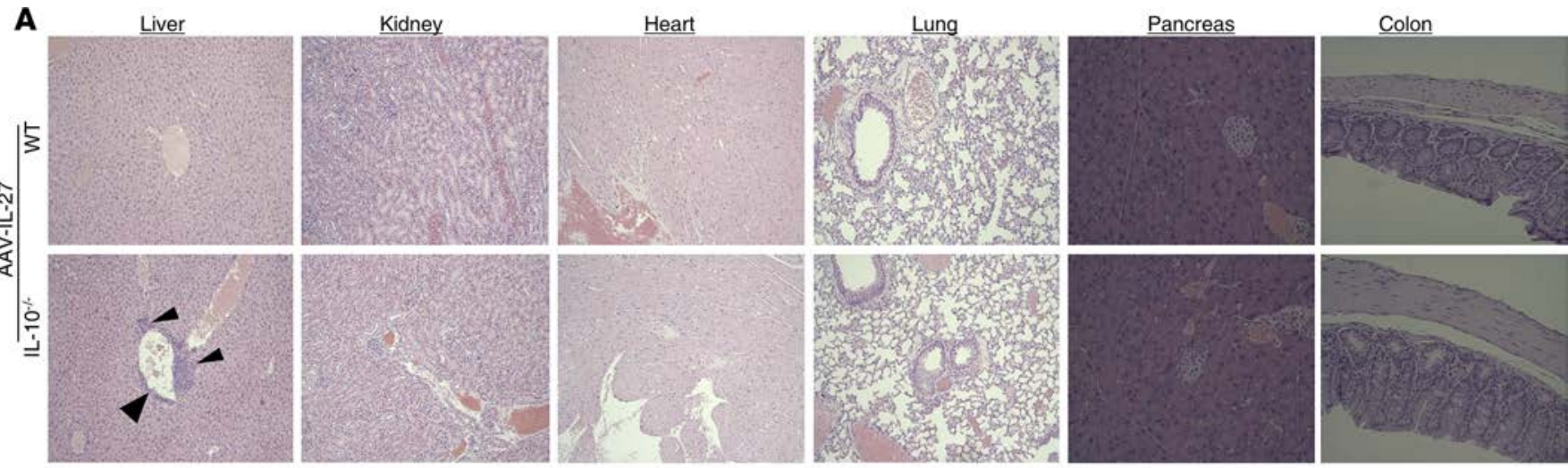

B
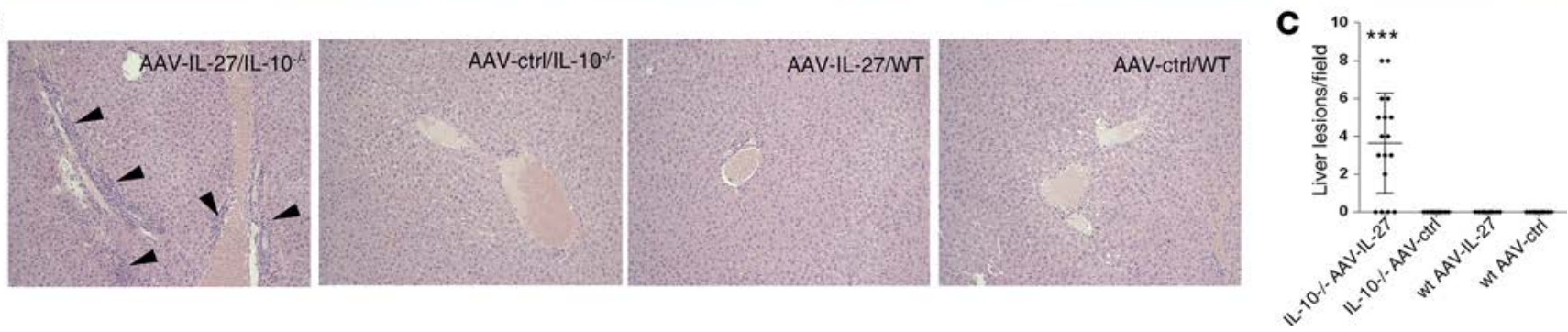

Figure 9. IL-10 prevents liver injury in AAV-IL-27-treated mice. (A) Representative histological images (H\&E) of various organs from AAV-IL-27-treated WT or IL-10-1- mice indicated in Figure 8D. AAV-IL-27-treated mice were sacrificed 2 months after treatment. Histological images of sections from various organs were examined and photographed using a microscope under $100 \times$ condition. Arrow heads indicate inflammatory lesions in liver tissue. (B and $\mathbf{C}$ ) $\mathrm{BALB} / \mathrm{C}$ and IL-10/-BALB/c mice were treated with AAV-IL-27 or AAV-ctrl virus i.m. Two months later, mice were sacrificed and representative histological images of livers from AAV-IL-27 or AAV-ctrl virus-treated WT or IL-10 $/-$ mice were shown (B). Arrow heads indicate inflammatory lesions in liver tissue. Numbers of inflammatory lesions in livers of AAV-treated mice were counted under the $100 \times$ field of a microscope and quantified (C). Dots represent the numbers obtained from individual microscopic fields. Five mice per group were used for this experiment. ${ }^{* *} P<0.001$ by Mann-Whitney $U$ test.

vitro studies. In systems where Tregs are exposed to excessive IL-2, IL-27 does not inhibit Treg homeostasis due to IL-2 signaling in Tregs counterreacting with the IL-27 signaling, as suggested by this study. Thus, we predict that Treg depletion by IL-27 could occur in settings where IL-27 level is high, while IL-2 signaling is low, which is the case in tumors. Because AAV-IL-27 therapy depletes Tregs while IL-2 signaling prevents IL-27-mediated Treg depletion, AAV-IL-27 and IL-2 combination therapy should be avoided, despite the fact that IL-27/IL-2 synergy in tumor immunotherapy has been reported (54).

Taken together, this study revealed new approaches of cancer immunotherapy by combining IL-27 administration and cancer vaccination or PD-1 blockade. The new approaches address all 3 restraining factors for cancer immunotherapy: First, through massive reduction of Tregs, active immune suppression during $\mathrm{T}$ cell activation and effector functions are removed, without causing significant adverse events. Second, by combination with cancer vaccines, we can optimally stimulate and expand tumor-specific T cells. Third, IL-27 therapy enhances cancer sensitivity to anti-PD-1 therapy by induction of T cell infiltration, PD-L1 expression, and Treg reduction, thereby rejuvenating $\mathrm{T}$ cells. Thus, our current study demonstrates the potential of AAV-IL-27 as an independent cancer therapeutic and efficient adjuvant for cancer vaccination or checkpoint blockade therapy.

\section{Methods}

Mice and tumor establishment. C57BL/6, CD45.1+C57BL6, BALB/c, IL10-/-BALB/c, IL10-/-C57BL/6, $\mathrm{Rag}^{-/-}$, and IL27 $\mathrm{ro}^{-/-}$mice were purchased from The Jackson Laboratory and were maintained in the animal facilities of the Ohio State University. Stat1 ${ }^{-/}$BALB/c mice have been described previously (55). B16.F10 melanoma, MC38 colon tumor, and EO771 breast tumor cells were originally obtained from ATCC and used after a few passages in vitro. Plasmacytoma J558 cells have been described previously $(23,24)$. These cancer cells were maintained in RPMI 1640 medium (Gibco) supplemented with $100 \mu \mathrm{g} /$ $\mathrm{ml}$ penicillin, $100 \mu \mathrm{g} / \mathrm{ml}$ streptomycin, and 5\% FBS (Gibco). To establish tumors in mice, indicated numbers of cancer cells were injected s.c. or intramammarily into mice in $100 \mu \mathrm{lof}$ PBS. The length (i) and width (ii) of tumors were measured using a digital caliper every 2 or 3 days. The tumor volume was cal- 
culated according to the formula volume $(V)=\mathrm{ab}^{2} / 2$, where a represents length and $\mathrm{b}$ represents width. To establish melanoma lung metastasis, each mouse was injected with $2 \times 10^{5}$ B16.F10 cells via the tail vein. Mice were monitored for up to 3-4 weeks. At the end of the experiments, mice were sacrificed, and their lungs were weighed and examined for tumor metastasis.

Production of rAAVs. rAAV-IL-27 and rAAV-IL-2 were produced as we previously described (48) or produced by Obio technology Corp. Briefly, IL-27 or IL-2 CDNA was inserted into an AAV carrier vector under the control of the CMV-chicken $\beta$-actin hybrid (CAG) promoter. The IL-27 carrier AAV vectors and the control vector were compacted with a $\mathrm{pAAV}-\mathrm{R} / \mathrm{C}$ vector (AAV serotype 8 [AAV8]) and an adenoviral helper vector in $293 \mathrm{~K}$ cells. Viruses were purified with $\mathrm{CsCl}$ gradient ultracentrifugation, and the titers of the generated AAV vectors were determined by real-time PCR. The generated AAV-IL-27, AAV-IL-2, and AAV-ctrl vectors were typically with titers of about $1 \times 10^{13} \mathrm{DRP} / \mathrm{ml}$. The generated AAV vectors were aliquoted and stored at $-80^{\circ} \mathrm{C}$ before use.

Treatment of mice with tumors using AAV. Cancer cells were injected into each C57BL6 or BALB/c mouse s.c. Four days later, mice were treated with AAV-IL-27 or AAV-ctrl vectors diluted in PBS in a total volume of $100 \mu \mathrm{l}$, containing $2 \times 10^{11} \mathrm{DRP}$ of vectors. Viral vectors were injected i.m. into 2 sites of hind legs. The sizes of tumors in each group of mice were measured over time using a digital caliper (Fowler). In some experiments, starting on day $4-5$, mice were also treated with $300 \mu \mathrm{g} /$ mouse of anti-PD-1 or an isotype-matched control antibody i.p. at 3-day intervals for up to 3-4 times. Anti-PD-1 antibody (RMP114) and an isotype-matched control antibody (anti-rat IgG2a; 2A3) were purchased from BioXcell. In some experiments, mice were bled and serum IL-27 levels were detected by ELISA (eBiosciences).

Generation of mixed BM chimeric mice and treatment with $A A V$ vectors and/or antibodies. We followed a well-established method as described (37) to generate BM chimeras. Briefly, BM cells from IL-27R $\alpha^{-/-}$mice $\left(\mathrm{CD} 45.2^{+}\right)$were mixed with BM cells from CD $45.1^{+} \mathrm{C} 57 \mathrm{BL} / 6$ mice at a $1: 1$ ratio, and $5 \times 10^{6}$ mixed BM cells were injected into each lethally irradiated (1,000 rads) C57BL/ 6 recipient. Twelve weeks after BM transfer, chimeric mice were injected with $0.2 \times 10^{6}$ B16.F10 cells per mouse s.c. and were treated with AAV-IL-27 or AAV-ctrl virus 4 days later.

Generation of B16-GM-CSF cells and mouse immunization. We generated a lentiviral vector expressing GMCSF by inserting GM-CSF cDNA into an expression vector (plvx-IRES-Zsgreen1; Clontech), which also expresses ZsGreen. Lentivirus expressing GM-CSF or the control Lenivirus (expressing ZsGreen only) was used to infect B16.F10 cells. The resulting cells were sorted based on their expression of ZsGreen using a FACS sorter (Mflo XDP, Beckman Coulter). The ZsGreen ${ }^{+}$cells that secrete GM-CSF (B16-GM-CSF) or not (B16-ctrl) were injected into mice s.c. for testing tumorigenicity. In some experiments, lethally irradiated (3,000 rads) B16-GM-CSF or B16-ctrl cells were injected into C57BL/6 mice i.p. at a dose of $0.2 \times 10^{6} /$ mouse. One week after immunization, mice were challenged with B16.F10 cells $\left(0.2 \times 10^{6} / \mathrm{mouse}\right)$ s.c. On day 4 after tumor cell challenge, mice were also treated with AAV-IL-27 or AAV-ctrl virus at a dose of $2 \times$ $10^{11} \mathrm{DRP} /$ mouse. Tumor growth and survival were observed as described above.

Flow cytometry. FITC-, PE-, APC-, or Percp-labeled antibodies to CD3 (17A2), CD4 (GK1.5), CD8 $\alpha$ (536.7), CD11b (M1/70), CD11c (N418), CD19 (6D5), CD25 (PC61), CD45 (30-F11), CD45.2 (104), NK1.1 (PK136), I-A/I-E (M5/114.15.2), PD-L1 (MIH5), FoxP3 (NRRF-30), IL-2 (JES6-5H4), IFN- $\gamma$ (XMG1.2), Granzyme B (GB11), and Perforin (S16009A) and isotype-matched control antibodies were purchased from BD Biosciences (antibodies to CD3, CD4, CD8, CD11b, CD11c, NK1.1, I-A/I-E, Granzyme B, Perforin, IL-2, IFN-g and control antibodies) or eBiosciences (antibodies to CD19, CD25, CD45, CD45.2, PD-L1, FoxP3 and control antibodies). For staining of cell-surface markers, disassociated cells from tumors were stained with various antibodies in staining buffer (PBS with 1\% FCS) and incubated on ice for 30 minutes. After washing with staining buffer, cells were fixed in 1\% paraformaldehyde in PBS. For intracellular cytokine staining, cells were stimulated in culture medium for 4 hours with $100 \mathrm{ng} / \mathrm{ml}$ of PMA and $500 \mathrm{ng} / \mathrm{ml}$ of ionomycin in the presence of GolgiStop (1:1,500; BD Biosciences). Viable cells were then fixed in IC fixation buffer (eBioscience), permeabilized with $1 \times$ permeabilization buffer (eBioscieces) and stained with respective antibodies to cytokines. Foxp3 staining was performed according to manufacturer's protocol (BD Biosciences). Stained cells were analyzed on a FACSCalibur flow cytometer, and data were analyzed using the flowjo software (Tree Star Inc.).

Peptide synthesis and IFN- $\gamma$ ELISPOT assay. Recently, the whole-genome sequencing of B16.F10 tumor cells identified 21 mutated tumor antigens (32). The mutated peptides were synthesized by GL Biochem Ltd. based on the sequences published by Kreiter et al (32). IFN- $\gamma$ ELISPOT assays were used to evaluate T cell responses to these peptide antigens. We used an IFN- $\gamma$ ELISPOT assay kit (Dakewe) and followed the protocol provided 
in the kit for the assay. Spleen cells from AAV-IL-27/B16-GM-CSF-treated tumor-bearing mice or controls were first depleted of antigen-presenting cells (APC) using rat anti-mouse CD16/CD32 mAb (2.4G2; BD Biosciences) and sheep anti-rat IgG Dyna beads (Thermo Fisher); then, the APC-depleted splenocytes were used as responders $\left(1 \times 10^{6} / \mathrm{ml}\right)$, and irradiated spleen cells from naive B6 mice were used as APC $\left(2 \times 10^{6} / \mathrm{ml}\right)$. Each peptide was used at a final concentration of $20 \mu \mathrm{g} / \mathrm{ml}$.

Histology. Mice were sacrificed by $\mathrm{CO}_{2}$ inhalation. Various organs were removed and fixed in $10 \%$ formalin/PBS. Paraffin sections were prepared and stained with H\&E in the histology core laboratory in the Department of Pathology and Comprehensive Cancer Center at the Ohio State University. Histological images were photographed using a microscope. Inflammatory lesions in liver sections were counted under a $100 \times$ field with a microscope, and 3 random fields were counted for each sample.

Statistics. Two-tailed Student's $t$ tests, 1-way ANOVA, and Mann-Whitney $U$ tests were used for statistical analyses. For comparison of mice survival, Kaplan-Meier survival analyses and log-rank tests were performed. The GraphPad Prism software was used for all the analyses. A $P$ value less than 0.05 was considered significant.

Study approval. All mice works involved in this study were approved by IACUC of the Ohio State University College of Medicine or Shanghai Jiao Tong University School of Medicine. The animal facilities were fully accredited by American Association for Accreditation of Laboratory Animal Care.

\section{Author contributions}

JZ, JQL, MS, XC, MD, and XFB performed experiments; JQL, JCZ, and JPD produced AAV vectors; SV and ARS provided Stat1-KO mice and helped in designing related experiments; LL helped with mice irradiation; XP helped with statistical analyses; XFB, PZ, and YL designed experiments and analyzed data; XFB wrote the manuscript.

\section{Acknowledgments}

This study is supported by grants from the NIH (CA198037) and China Natural Science Foundation (81572805).

Address correspondence to: Xue-Feng Bai, Department of Pathology and Comprehensive Cancer Center, Ohio State University Medical Center, 129 Hamilton Hall, 1645 Neil Avenue, Columbus, Ohio 43210, USA. Phone: 614.292.8649; Email: Xue-Feng.Bai@osumc.edu.

1. Boon T, Cerottini JC, Van den Eynde B, van der Bruggen P, Van Pel A. Tumor antigens recognized by T lymphocytes. Annu Rev Immunol. 1994;12:337-365.

2. Lee PP, et al. Characterization of circulating T cells specific for tumor-associated antigens in melanoma patients. Nat Med. 1999;5(6):677-685.

3. Romero P, et al. Ex vivo staining of metastatic lymph nodes by class I major histocompatibility complex tetramers reveals high numbers of antigen-experienced tumor-specific cytolytic T lymphocytes. J Exp Med. 1998;188(9):1641-1650.

4. Rosenberg SA, Yang JC, Restifo NP. Cancer immunotherapy: moving beyond current vaccines. Nat Med. 2004;10(9):909-915.

5. Ott PA, et al. An immunogenic personal neoantigen vaccine for patients with melanoma. Nature. 2017;547(7662):217-221

6. Sahin U, et al. Personalized RNA mutanome vaccines mobilize poly-specific therapeutic immunity against cancer. Nature. 2017;547(7662):222-226.

7. Curiel TJ, et al. Specific recruitment of regulatory $\mathrm{T}$ cells in ovarian carcinoma fosters immune privilege and predicts reduced survival. Nat Med. 2004;10(9):942-949.

8. Onizuka S, Tawara I, Shimizu J, Sakaguchi S, Fujita T, Nakayama E. Tumor rejection by in vivo administration of anti-CD25 (interleukin-2 receptor alpha) monoclonal antibody. Cancer Res. 1999;59(13):3128-3133.

9. Zhou P, Zheng X, Zhang H, Liu Y, Zheng P. B7 blockade alters the balance between regulatory T cells and tumor-reactive T cells for immunotherapy of cancer. Clin Cancer Res. 2009;15(3):960-970.

10. Simpson TR, et al. Fc-dependent depletion of tumor-infiltrating regulatory T cells co-defines the efficacy of anti-CTLA-4 therapy against melanoma. J Exp Med. 2013;210(9):1695-1710

11. Selby MJ, et al. Anti-CTLA-4 antibodies of IgG2a isotype enhance antitumor activity through reduction of intratumoral regulatory T cells. Cancer Immunol Res. 2013;1(1):32-42.

12. Phan GQ, et al. Cancer regression and autoimmunity induced by cytotoxic T lymphocyte-associated antigen 4 blockade in patients with metastatic melanoma. Proc Natl Acad Sci USA. 2003;100(14):8372-8377.

13. Hamid O, et al. Safety and tumor responses with lambrolizumab (anti-PD-1) in melanoma. N Engl J Med. 2013;369(2):134-144.

14. Topalian SL, et al. Safety, activity, and immune correlates of anti-PD-1 antibody in cancer. NEngl J Med. 2012;366(26):2443-2454.

15. Topalian SL. Targeting Immune Checkpoints in Cancer Therapy. JAMA. 2017;318(17):1647-1648.

16. Lucas S, Ghilardi N, Li J, de Sauvage FJ. IL-27 regulates IL-12 responsiveness of naive CD4+ T cells through Stat1-dependent and -independent mechanisms. Proc Natl Acad Sci USA. 2003;100(25):15047-15052.

17. Diveu C, et al. IL-27 blocks RORc expression to inhibit lineage commitment of Th17 cells. J Immunol. 2009;182(9):5748-5756. 
18. Shinozaki Y, et al. Tumor-specific cytotoxic $\mathrm{T}$ cell generation and dendritic cell function are differentially regulated by interleukin 27 during development of anti-tumor immunity. Int J Cancer. 2009;124(6):1372-1378

19. Dibra D, et al. Lack of Immunomodulatory Interleukin-27 Enhances Oncogenic Properties of Mutant p53 In Vivo. Clin Cancer Res. 2016;22(15):3876-3883.

20. Hisada M, et al. Potent antitumor activity of interleukin-27. Cancer Res. 2004;64(3):1152-1156.

21. Salcedo R, et al. IL-27 mediates complete regression of orthotopic primary and metastatic murine neuroblastoma tumors: role for CD8+ T cells. J Immunol. 2004;173(12):7170-7182.

22. Zhu S, Lee DA, Li S. IL-12 and IL-27 sequential gene therapy via intramuscular electroporation delivery for eliminating distal aggressive tumors. J Immunol. 2010;184(5):2348-2354.

23. Liu Z, Liu JQ, Talebian F, Wu LC, Li S, Bai XF. IL-27 enhances the survival of tumor antigen-specific CD8+ T cells and programs them into IL-10-producing, memory precursor-like effector cells. Eur J Immunol. 2013;43(2):468-479.

24. Liu Z, et al. Epstein-Barr virus-induced gene 3-deficiency leads to impaired antitumor T-cell responses and accelerated tumor growth. Oncoimmunology. 2015;4(7):e989137.

25. Yoshimoto T, et al. Potential clinical application of interleukin-27 as an antitumor agent. Cancer Sci. 2015;106(9):1103-1110.

26. Aalbers CJ, Tak PP, Vervoordeldonk MJ. Advancements in adeno-associated viral gene therapy approaches: exploring a new horizon. F1000 Med Rep. 2011;3:17.

27. Sereti I, Gea-Banacloche J, Kan MY, Hallahan CW, Lane HC. Interleukin 2 leads to dose-dependent expression of the alpha chain of the IL-2 receptor on CD25-negative T lymphocytes in the absence of exogenous antigenic stimulation. Clin Immunol. 2000;97(3):266-276.

28. Villarino AV, Stumhofer JS, Saris CJ, Kastelein RA, de Sauvage FJ, Hunter CA. IL-27 limits IL-2 production during Th1 differentiation. J Immunol. 2006;176(1):237-247.

29. Owaki T, et al. IL-27 suppresses CD28-mediated [correction of medicated] IL-2 production through suppressor of cytokine signaling 3. J Immunol. 2006;176(5):2773-2780.

30. Colombo MP, Piconese S. Regulatory-T-cell inhibition versus depletion: the right choice in cancer immunotherapy. Nat Rev Cancer. 2007;7(11):880-887.

31. Zhou G, Drake CG, Levitsky HI. Amplification of tumor-specific regulatory T cells following therapeutic cancer vaccines. Blood. 2006;107(2):628-636.

32. Kreiter S, et al. Mutant MHC class II epitopes drive therapeutic immune responses to cancer. Nature. 2015;520(7549):692-696.

33. Hirahara K, et al. Interleukin-27 priming of T cells controls IL-17 production in trans via induction of the ligand PD-L1. Immunity. 2012;36(6):1017-1030.

34. Takeda A, et al. Cutting edge: role of IL-27/WSX-1 signaling for induction of T-bet through activation of STAT1 during initial Th1 commitment. J Immunol. 2003;170(10):4886-4890.

35. Awasthi A, et al. A dominant function for interleukin 27 in generating interleukin 10-producing anti-inflammatory T cells. Nat Immunol. 2007;8(12):1380-1389.

36. Wang L, Liu JQ, Talebian F, Liu Z, Yu L, Bai XF. IL-10 enhances CTL-mediated tumor rejection by inhibiting highly suppressive CD4+T cells and promoting CTL persistence in a murine model of plasmacytoma. Oncoimmunology. 2015;4(7):e1014232.

37. Wojno ED, et al. A role for IL-27 in limiting T regulatory cell populations. J Immunol. 2011;187(1):266-273.

38. Babon JJ, Varghese LN, Nicola NA. Inhibition of IL-6 family cytokines by SOCS3. Semin Immunol. 2014;26(1):13-19.

39. Hennighausen L, Robinson GW. Interpretation of cytokine signaling through the transcription factors STAT5A and STAT5B. Genes Dev. 2008;22(6):711-721.

40. Pillemer BB, Xu H, Oriss TB, Qi Z, Ray A. Deficient SOCS3 expression in CD4+CD25+FoxP3+ regulatory T cells and SOCS3-mediated suppression of Treg function. Eur J Immunol. 2007;37(8):2082-2089.

41. Dranoff G, et al. Vaccination with irradiated tumor cells engineered to secrete murine granulocyte-macrophage colony-stimulating factor stimulates potent, specific, and long-lasting anti-tumor immunity. Proc Natl Acad Sci USA. 1993;90(8):3539-3543

42. Tumeh PC, et al. PD-1 blockade induces responses by inhibiting adaptive immune resistance. Nature. 2014;515(7528):568-571

43. Lin H, et al. Host expression of PD-L1 determines efficacy of PD-L1 pathway blockade-mediated tumor regression. J Clin Invest. 2018;128(2):805-815.

44. Ngiow SF, et al. A Threshold Level of Intratumor CD8+ T-cell PD1 Expression Dictates Therapeutic Response to Anti-PD1. Cancer Res. 2015;75(18):3800-3811.

45. Attia P, Maker AV, Haworth LR, Rogers-Freezer L, Rosenberg SA. Inability of a fusion protein of IL-2 and diphtheria toxin (Denileukin Diftitox, DAB389IL-2, ONTAK) to eliminate regulatory T lymphocytes in patients with melanoma. J Immunother. 2005;28(6):582-592.

46. Kim JM, Rasmussen JP, Rudensky AY. Regulatory T cells prevent catastrophic autoimmunity throughout the lifespan of mice. Nat Immunol. 2007;8(2):191-197.

47. Stumhofer JS, et al. Interleukins 27 and 6 induce STAT3-mediated T cell production of interleukin 10. Nat Immunol. 2007;8(12):1363-1371

48. Zhu X, et al. Systemic delivery of IL-27 by an adeno-associated viral vector inhibits T cell-mediated colitis and induces multiple inhibitory pathways in T cells. J Leukoc Biol. 2016;100(2):403-411.

49. Naing A, et al. Safety, Antitumor Activity, and Immune Activation of Pegylated Recombinant Human Interleukin-10 (AM0010) in Patients With Advanced Solid Tumors. J Clin Oncol. 2016;34(29):3562-3569.

50. Neufert C, et al. IL-27 controls the development of inducible regulatory T cells and Th17 cells via differential effects on STAT1. Eur J Immunol. 2007;37(7):1809-1816.

51. Hall AO, et al. The cytokines interleukin 27 and interferon- $\gamma$ promote distinct Treg cell populations required to limit infectioninduced pathology. Immunity. 2012;37(3):511-523.

52. Do J, et al. Treg-specific IL-27R $\alpha$ deletion uncovers a key role for IL-27 in Treg function to control autoimmunity. Proc Natl Acad Sci USA. 2017;114(38):10190-10195

53. Do JS, et al. An IL-27/Lag3 axis enhances Foxp3+ regulatory T cell-suppressive function and therapeutic efficacy. Mucosal Immunol. 2016;9(1):137-145. 
54. Salcedo R, et al. Immunologic and therapeutic synergy of IL-27 and IL-2: enhancement of T cell sensitization, tumor-specific CTL reactivity and complete regression of disseminated neuroblastoma metastases in the liver and bone marrow. J Immunol. 2009;182(7):4328-4338.

55. Liu Z, et al. Interleukin-27 signalling induces stem cell antigen-1 expression in $\mathrm{T}$ lymphocytes in vivo. Immunology. 2017;152(4):638-647. 